Manuscript title: Berth allocation and quay crane assignment for the trade-off between service efficiency and operating cost considering carbon emission taxation

First Author (Corresponding author):

Tingsong Wang,

School of Economics and Management,

Wuhan University

Wuhan 430072, P.R.China

E-mail: emswangts@whu.edu.cn

Second Author:

Yuquan Du,

Department of Maritime and Logistics Management,

Australian Maritime College,

University of Tasmania, Launceston TAS 7250, Australia

Tel.: +61 036324 3123;

E-mail: yuquan.du@utas.edu.au

Third Author

Debin Fang,

School of Economics and Management,

Wuhan University

Wuhan 430072, P.R.China

E-mail: dbfang@whu.edu.cn

Fourth Author

Zhichun Li,

School of Management,

Huazhong University of Science and Techonology

Wuhan 430072, P.R.China

E-mail:smzcli@gmail.com 


\title{
Berth allocation and quay crane assignment for the trade-off between service efficiency and operating cost considering carbon emission taxation
}

\begin{abstract}
Under the requirement of energy saving and emission reduction, green ports have been a focus to sustain the development of maritime transportation. It's reported that the International Maritime Organization (IMO) has proposed to impose carbon emission tax on ports in the long term, which would definitely increase the operating cost of ports. Quay cranes (QCs), as one type of handling equipment, plays an important role in the service efficiency and carbon emission of ports. Therefore, this paper makes effort to explore the study of integrated berth allocation and QC assignment problem with the consideration of carbon emission taxation. This problem is formulated as a biobjective integer programming model, aiming at minimizing the total completion delay of all tasks and the total operating costs for all QCs. Finally, numerical experiments are performed to assess the applicability of the proposed models and evaluate the efficiency of the developed solution algorithm.
\end{abstract}

Keywords: OR in maritime industry; berth allocation and quay crane assignment; carbon emission taxation; biobjective integer programming; balanced box method.

\section{Introduction}

Ports are a key node in the global maritime transportation network. Under the requirement of energy saving and emission reduction, the carbon emission of port area is highly concerned by governments and port operators. Recently, initiatives of green ports have been proposed by more and more governments and port operators, such as the "Clean Air Action Plan" proposed by Port of Long Beach and Port of Los Angeles (Jelenić, 2016), the "Clean Air Action Program" developed by Port of Rotterdam (Mshe, 2012), and the "Clean Air Strategy" for Port of New York \& New Jersey (Port of NY \& NJ, 2009). Besides those plans to construct green ports, the Maritime Environmental Protection Committee (MEPC) under International Maritime Organization (IMO) proposes to impose carbon emission tax on ports in the long term (IMO, 2005). If the policy of carbon taxation is implemented, it will definitely increase the operating cost of ports. It is noted that the carbon emission at port to be taxed refers to that emitted from cargo handling equipment. Though some cargo handling equipment may use electric power in some advanced container terminals, such as quay cranes (QCs) in Waigaoqiao Terminal and Yangshan Terminal in Shanghai Port, the consumption of electricity would indirectly produce carbon emissions as well. Geerlings and Duin (2011) estimate the carbon emissions 
due to the operations of electric QCs for the Port of Rotterdam. Therefore, the port operators are facing the pressure on increment of operating costs due to payment of carbon emission tax.

Meanwhile, the fierce market competition among ports makes port operators have to improve their service efficiency to attract more customers, especially under the current environment of depressed global economics. The service efficiency is related to the QC hours to complete the work tasks. It is noted that the required QC-hours depend on the quantity of workload of vessels moored at ports and the productivity of QCs. The productivity of QCs depends on the hoisting speed to lift containers and the trolley speed to transversely move containers between vessels and shore platform. The productivity of stevedoring also varies because of interference among QCs and deviation of the vessel from its desired berthing position (Schonfeld and Sharafeldien, 1985; Meisel and Bierwirth, 2009). Poor berthing and QC allocation have negative effect on the productivity of stevedoring, which result in more QC-hours, indicating more carbon missions and lower service efficiency of ports.

Therefore, the port operator should optimize the berthing allocation and QC assignment jointly to improve the service efficiency of the port and save the total operating costs with the consideration of carbon emission taxation. However, the improvement of service efficiency cannot be fulfilled without incurring additional operating costs. Thus, the goal of the port operator at the operational level is to seek the optimal trade-off between cost saving and service efficiency improvement, which is the focus of this study.

This study makes the following substantial contributions:

1) This paper introduces the carbon emission taxation levied on ports to the classical berth allocation and quay crane assignment problems, and formulates the proposed problem as a biobjective integer programming (BOIP) model for the trade-off between service efficiency and operating costs of port operators, which represents our main contribution to the literature on port operations.

2) The nonlinearity of the objective functions in the BOIP model caused by the piecewise taxation rates of carbon emission increases the hardness of this model. To reduce the hardness, a linearization technique is applied. Furthermore, an efficient solution algorithm is designed to solve it based on the analysis of the structure and properties of the BOIP model, whose efficiency and effectiveness are shown by numerical experiments.

3) We investigate the effect of economic analysis on port operating costs and environmental 
analysis on carbon emission. We find that the introducing carbon emission taxation on port can reduce the carbon emission from QCs, which indicates that it benefits the improvement of environment at port; but the level of service efficiency of port decreases. Computational results reveal the trade-off between carbon emission and port service efficiency. Therefore, the port operator can improve service efficiency at the expense of carbon emission, and vice versa.

The remainder of this paper is organized as follows. Section 2 reviews the existing literature and summarizes their limitations. Section 3 addresses the integrated berth allocation and quay crane assignment problem, and analyzes the trade-off between port service efficiency and operating costs. The notations used in this paper and the BOIP model are described in Section 4. Section 5 elaborates on the solution algorithm to solve the BOIP model. Section 6 conducts extensive numerical experiments to validate the effectiveness of the proposed model and the efficiency of the proposed solution algorithm. Section 7 concludes this study.

\section{Literature review}

There have been numerous studies on berth allocation and QC assignment problems in container terminals (see the literature review papers: Bierwirth and Meisel, 2010, 2015). Most of these studies are devoted to promote the handling efficiency by separately optimizing berth allocation or QC assignment, whereas fewer studies jointly consider them both. As for the problems of carbon emission of QCs, only a small number of studies can be found. This section firstly reviews the studies highly related to integrated berth allocation and QC assignment problem, and then, a review of studies related to carbon emission of QCs is stated.

\subsection{The integrated berth allocation and QC assignment problems}

The integrated berth allocation and QC assignment problem (BAQCAP) is firstly studied by Park and Kim (2003), and it is formulated as a two-phase integer programing model. Imai et al. (2008) propose a genetic algorithm (GA) to seek an approximate solution for the BAQCAP without evaluating the solution quality. Later, Chang et al. (2010) reconsider the BAQCAP, and formulate it as a dynamic allocation model based on a rolling horizon approach. They design a heuristic to solve the proposed model. Giallombardo et al. (2010) develop a heuristic based on tabu search to solve the BAQCAP, and evaluate the superiority of the proposed heuristic solution algorithm. Shang et al. (2016) formulate the BAQCAP as a robust optimization model, and develop a GA and an insertion 
heuristic algorithm to solve the model. It is noted that all the solution algorithms reviewed above are not exact. To design an exact algorithm for the BAQCAP, Vacca et al. (2013) exploit the problem structure, and propose a branch-and-price algorithm based on column generation. Zhen et al. (2017) propose a more practical BAQCAP by taking the tides and channel flow control constraints into account. Agra and Oliveira (2018) use the rolling horizon heuristic to derive good feasible solutions for BAQCAP.

However, the productivity of QCs considered in all the literature mentioned above is implicitly assumed to be immutable, which may yield biased results for decision-makers because the productivity of QCs is affected by the interference among QCs and deviation of the vessel from its desired berthing position (Schonfeld and Sharafeldien, 1985; Meisel and Bierwirth, 2009). With consideration of the factors affecting the productivity of QCs, Meisel and Bierwirth (2009) reformulate the BAQCAP, and propose two meta-heuristics to solve it. Based on the model proposed in Meisel and Bierwirth (2009), Li et al. (2015) propose a more practical BAQCAP by considering QC coverage range, and formulate it as a nonlinear mixed-integer programming model. A novel heuristic algorithm based on spatiotemporal conflict analysis is designed to obtain a high-quality solution to the model. Iris et al. (2015) extend the model in Meisel and Bierwirth (2009), and formulate the BAQCAP as two novel set partitioning models based on time-variant and invariant QC assignment policies, respectively. Continuously, Iris et al. (2017) improve their model and design an adaptive large neighborhood search heuristic for the BAQCAP.

It can be seen from the section above that none of these researches take energy consumption of QCs into account. Recently, He (2016) fills the gap, and formulates the proposed BAQCAP as a biobjective mixed-integer programming (BOMIP) model with the aim of minimizing the total departure delay of all vessels and the total handling energy consumption of all QCs. It is noted that though He (2016) considers the energy consumption of all QCs, there are three differences between the work of He (2016) and ours: 1) Our study introduces the carbon emission taxation into the classical BAQCAP, which contributes a new research topic to the literature. Meanwhile, we provide a preliminary study to the initiative of carbon emission taxation imposing on port proposed by IMO. 2) The carbon emission tax in our study is a piecewise function with respect to the quantity of carbon emission, which causes the nonlinearity of the objective function in our model, and we provide a method to linearize it. However, the cost of energy consumed by QCs in He (2016) is a linear function 
with respect to the quantity of energy consumption, and his model is linear. Therefore, we actually improve He (2016) from the perspective of research problem and research methodology. 3) We design an efficient solution algorithm by exploiting the structure of our problem, while the solution algorithm of $\mathrm{He}$ (2016) cannot cope with the differences in our model, especially the computational complicatedness caused by the piecewise function in our study.

\subsection{The problems of carbon emission of QCs}

The studies related to the carbon emission issues of apron-side operations at a container terminal are quite limited. To the best of our knowledge, a total of four studies are found, in which two studies are on carbon emission of vessels (Du et al., 2011; Hu et al., 2014), and two studies are on carbon emission of QCs (Geerlings and Duin, 2011; Wang et al., 2018b). Geerlings and Duin (2011) analyze the sources of carbon emissions produced at container terminals, and calculate the carbon emissions generated by all container handling equipment at the container terminals of Port of Rotterdam. Wang et al. (2018b) propose the BAQCAP problem under different taxation policies for carbon emission. We have to highlight the differences between the work of Wang et al. (2018b) and ours: 1) Wang et al. (2018b) formulate the proposed problem as a single-objective integer optimization model to minimize the total costs, which contain the penalty costs of berth waiting and departure delay of all vessels, the operating costs of QCs, and carbon emission taxation of QCs. 2) As the model formulated in Wang et al. (2018b) is a single-objective integer optimization model, the branch-and-bound solution algorithm designed in Wang et al. (2018) cannot be used to solve our BOIP model. 3) The focus of our study is to seek the optimal trade-off between cost saving and service efficiency improvement, but Wang et al. (2018b) is to seek the optimal berth-QC plan for port operators.

To summarize, few of the above-reviewed studies incorporate the carbon emission taxation into the BAQCAP, although MEPC has advocated imposing the carbon emission taxation on ports in the long run. Therefore, our paper addresses the BAQCAP with explicit consideration of carbon emission taxation from the point of view of a port operator for the trade-off between service efficiency and operating costs. More specifically, this work formulates the BAQCAP as a BOIP model with aiming at maximizing the service efficiency (namely minimizing the berth waiting time and departure delay of all vessels) and minimizing the total operating costs of QCs.

\section{Problem description}

\subsection{Berth allocation and QC assignment}


We consider a container terminal and the cargo unit carried on each vessel is assumed to be $20-\mathrm{ft}$ equivalent unit (TEU). Given a set of container vessels (here, a vessel is denoted by index $i$ and the set of vessels is denoted by $\mathcal{V}:=\{1, \ldots, i, \ldots V\}$, the integrated BAQCAP aims at assigning a berthing position and a quay crane assignment profile to each vessel over a given time horizon as well as at scheduling incoming vessels according to their time windows. A QC profile for a vessel specifies the number of QCs serving this vessel in each time-unit during its stevedoring. It is noted that the QCs are assumed homogeneous in this paper. An example of an integrated BAQCAP plan in Figure 1 illustrates the berthing plan in a space-time diagram. In this example, a set of vessels are about to moor at the container terminal. Each vessel is represented by a rectangle that shows the berthing position and the time duration occupied by the vessel. The point $x_{i}$ on the horizontal axis represents the berthing position of vessel $i(i \in \mathcal{V})$, and the points $t_{i}^{a}, t_{i}^{b}, t_{i}^{d}$ and $\tilde{t}_{i}^{d}$ on vertical axis represents the arrival time, berthing time, estimated departure time and actual departure time of vessel $i(i \in \mathcal{V})$, respectively. Figure 1 shows the QC profile of each vessel as well. As can be seen from Figure 1, vessel $i(i \in \mathcal{V})$ is serviced for six time-units. In both of the first and second time-unit, five QCs are assigned to vessel $i(i \in \mathcal{V})$, and three QCs are assigned for each of the rest four time-units.

$<$ Figure 1 is inserted here $>$

\subsection{Port service efficiency analysis}

When a vessel $i(i \in \mathcal{V})$ is about to moor at the container terminal, it reports its expected arrival time and expected departure time to the port operator, denoted by $t_{i}^{a}$ and $t_{i}^{d}$, respectively. Accordingly, the port operator makes a berthing plan for the vessel $i$ which involves berthing position and berthing time, denoted by $x_{i}$ and $t_{i}^{b}$, respectively. If the vessel's expected arrival time $t_{i}^{a}$ is earlier than its berthing time $t_{i}^{b}$, i.e. $t_{i}^{a}<t_{i}^{b}$, it has to wait at the anchorage point until the berth space is available. The time interval between arrival time $t_{i}^{a}$ and berthing time $t_{i}^{b}$ is referred to as the berth waiting time, which is a performance indicator to evaluate the service efficiency of the port. Another performance indicator to evaluate the service efficiency of the port is departure delay time, which refers to as the time interval between the actual departure time $\tilde{t}_{i}^{d}$ and the required departure time $t_{i}^{d}$ when delay occurs, i.e. $t_{i}^{d}<\tilde{t}_{i}^{d}$. In order to maintain a good level of service for vessels, the port operator tries to avoid the occurrence of berth waiting and departure delay, which means that the minimization of berth waiting and departure delay is an essential aim for the port 
operator.

\subsection{Port operating costs analysis}

Here, the cost of operating QCs to load/unload containers is the fundamental cost to the port operator in the BAQCAP, which mainly includes the fuel consumption cost and labor cost. Recall that the QCs will be taxed under IMO's carbon emission taxation policies, which creates cost (tax) to the port operator as well. It's noted that thought QCs in some ports may use electricity as power, they are still to be levied tax because carbon tax is a form of pollution tax based on the economic principle of negative externalities, which are costs generated by the production of goods and services. Obviously, to generate the electricity used by QCs, fossil fuels and/or coal have to be burnt. Consequently, the burning creates carbon emission, which is pollution that has a societal cost, but not paid for yet. Therefore, the total cost of the port operator consists of the fundamental cost of operating QCs to handle containers, and the carbon emission taxation cost incurred by operating QCs. Obviously, the carbon emission taxation cost will increase the total operating costs for the port operator. Thus, saving the total operating costs is another essential aim for the port operator.

\subsection{Trade-off between service efficiency and operating costs}

The QC hours required to complete the workload depends on the workload of moored vessels and the productivity of QCs. It is found that the productivity of QCs is strongly affected by interference among QCs (Meisel and Bierwirth, 2009; Schonfeld and Sharafeldien, 1985). Given an interference exponent $\tilde{\alpha}(0<\tilde{\alpha} \leq 1)$, the productivity obtained from assigning $n$ QCs to a vessel for one hour is given by a total of $n^{\widetilde{\alpha}}$ QC-hours, which indicates that the interference among QCs harms the productivity of QCs, resulting in more working hours required to complete the workload. However, more QCs assignment to a vessel may let the vessel depart on the expected schedule, namely no departure delay occurs. For example, we assume that a vessel's expected mooring time at a port are five hours and the workload needs eight QC-hours if no interference among QCs. The port operator can make two QC assignment plans, A and B, for the vessel based on different preference. If the port service efficiency is preferred, then Plan A is to assign two QCs to the vessel and each QC works for five hours; if the saving total operating costs is preferred, then Plan B is to assign one QC to the vessel but needs to work for eight hours. Therefore, Plan A guarantees a good service efficiency but with more operating costs, while Plan B saves operating costs but with poor service efficiency. Therefore, the improvement of service efficiency may not be fulfilled without incurring additional operating 
costs. We need to seek a trade-off between the service efficiency and operating costs saving.

\section{Model development}

In this section, we firstly address the rate function of carbon emission taxation, and then, continue to develop a BOIP model to formulate the BAQCAP for the trade-off between service efficiency and operating cost saving with carbon emission taxation.

\subsection{Rate function of carbon emission taxation}

Here we consider a stepwise linear function for the carbon emission taxation rate, as stepwise tax rate is a quite general form in taxation theory (Schaefer, 1969), and it is widely applied in practice, like electricity tax rate levied in German (Flues and Lutz, 2014), etc. The carbon emission taxation rate function is denoted by $r(z)$, where $z$ is the amount of carbon emission in the period of study. Usually, the taxation rate increases in practice, but it may not be always like that. We found that the electricity tax rate decreases in German because the Germany government is concerned that the electricity tax may harm the competitiveness of German firms that are subject to competition from abroad (Flues and Lutz, 2014). Therefore, the carbon taxation rate function $r(z)$ in this paper is assumed to be a general form, neither increasing nor decreasing with increase of the amount of carbon emission $z$.

We divide the amount of carbon emission into $N$ segments and the dividing points are denoted by $\Gamma_{1}, \Gamma_{2}, \ldots, \Gamma_{\mathrm{N}-1}$ and $\Gamma_{N}\left(\Gamma_{N}:=\infty\right)$. Let $r_{1}, r_{2}, \ldots, r_{N}$ be taxation rate for the $1^{\text {st }}, 2^{\text {nd }}, \ldots, N^{\text {th }}$ segment of carbon emission, respectively. Figure 2 illustrates an example of the stepwise taxation rate function with three segments.

$<$ Figure 2 is inserted here $>$

Define $C^{C T}(z):=r(z) z$ as the total tax paid if the carbon emission is $z$. Since $r(z)$ is a stepwise function, $C^{C T}(z)$ is a piecewise linear function. Figure 3 illustrates the example of $C^{C T}(z)$ with three segments, in which $r_{1}, r_{2}, r_{3}$ are slopes for the $1^{\text {st }}, 2^{\text {nd }}, 3^{\text {rd }}$ segment, and $b_{1}, b_{2}, b_{3}$ are the corresponding intercepts.

$<$ Figure 3 is inserted here $>$

Carbon emission tax function $C^{C T}(z)$ is represented by Eq. (1) 


$$
C^{C T}(z)= \begin{cases}z \times r_{1} & \text { if } z \in\left[0, \Gamma_{1}\right) \\ z \times r_{2}+b_{2} & \text { if } z \in\left[\Gamma_{1}, \Gamma_{2}\right) \\ M & \text { if } z \in\left[\Gamma_{N-1}, \Gamma_{N}\right)\end{cases}
$$

where intercepts $b_{1}, b_{2}, \ldots, b_{N}$ are given by Proposition 1 .

Proposition 1. For $s=2, \ldots, N$, we have $b_{s}=\sum_{j=1}^{s-1} \Gamma_{j}\left(r_{j}-r_{j+1}\right)$.

Proof: If the amount of carbon emission $z$ is located in the $s^{\text {th }}$ segment $(s=2, \ldots, N)$, namely, $z \in$ $\left[\Gamma_{s-1}, \Gamma_{s}\right)$, then the carbon emission taxation cost function is given by:

$$
\begin{aligned}
C^{C T} & \left(z \mid z \in\left[\Gamma_{s-1}, \Gamma_{s}\right)\right) \\
& =\Gamma_{1} r_{1}+\left(\Gamma_{2}-\Gamma_{1}\right) r_{2}+\left(\Gamma_{3}-\Gamma_{2}\right) r_{3}+\ldots+\left(\Gamma_{s-1}-\Gamma_{s-2}\right) r_{s-1}+\left(z-\Gamma_{s-1}\right) r_{s} \\
& =\Gamma_{1}\left(r_{1}-r_{2}\right)+\Gamma_{2}\left(r_{2}-r_{3}\right)+\ldots+\Gamma_{s-2}\left(r_{s-2}-r_{s-1}\right)+\Gamma_{s-1}\left(r_{s-1}-r_{s}\right)+z r_{s} \\
& =\sum_{j=1}^{s-1} \Gamma_{j}\left(r_{j}-r_{j+1}\right)+z r_{s}
\end{aligned}
$$

According to Eq. (1), the carbon emission tax with $z \in\left[\Gamma_{s-1}, \Gamma_{s}\right)$ can be calculated by $C^{C T}\left(z \mid z \in\left[\Gamma_{s-1}, \Gamma_{s}\right)\right)=b_{s}+z r_{s}$, where $b_{s}=\sum_{j=1}^{s-1} \Gamma_{j}\left(r_{j}-r_{j+1}\right)$.

\subsection{The BOIP model}

Before we develop the BIOP model for the BAQCAP, we firstly introduce the notations used in our model, which is presented below.

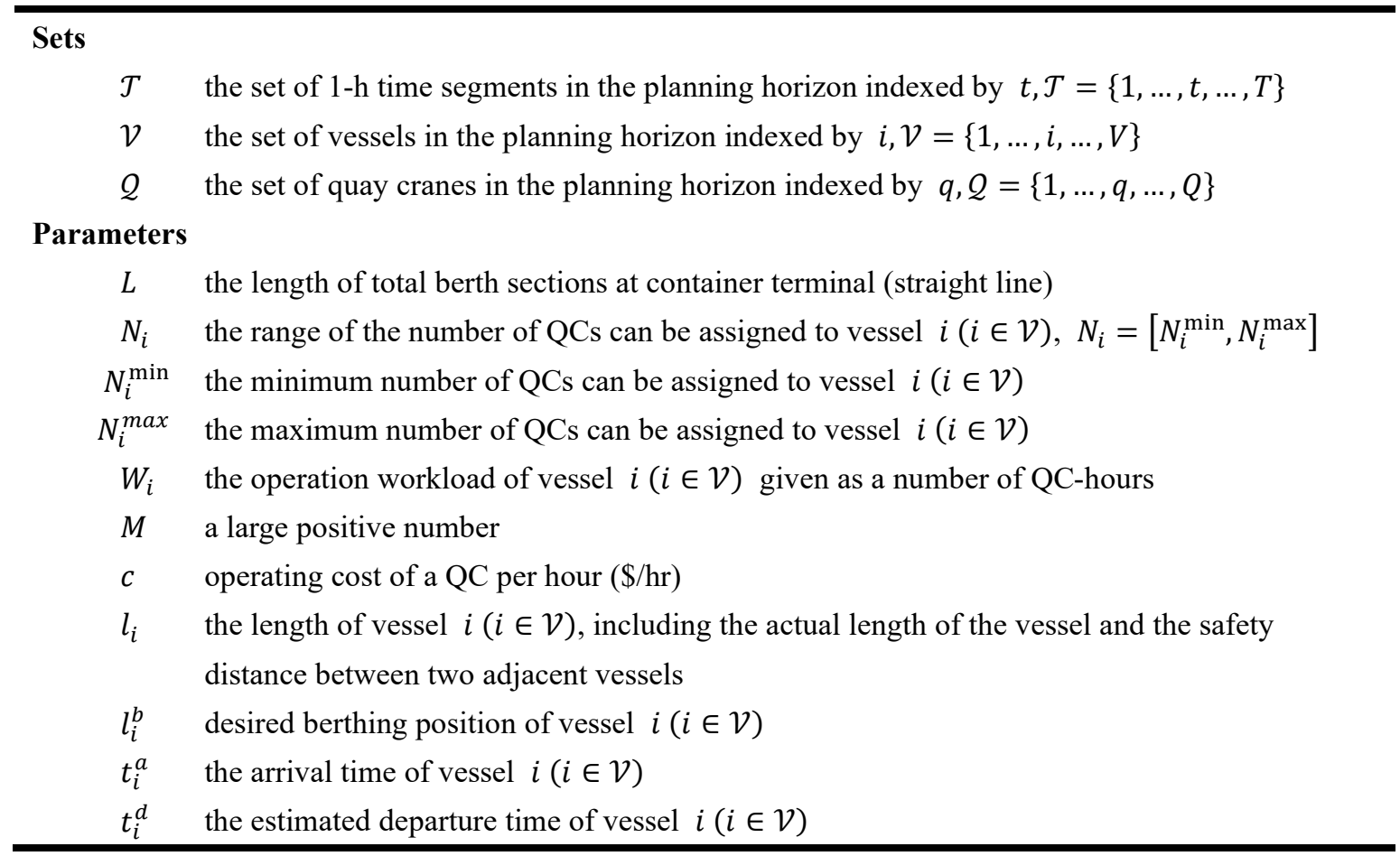


$\mu \quad$ the energy consumption of a QC during a unit time segment (unit: $\mathrm{kWh} / \mathrm{hr}$ )

$\alpha \quad$ carbon emission factor for a QC (unit: $\mathrm{kg} / \mathrm{kWh}$ )

$\tilde{\alpha} \quad$ interference factor of QCs operation

$\hat{\alpha} \quad$ berth deviation factor

\section{Decision variables}

$x_{i} \quad$ berthing position of vessel $i(i \in \mathcal{V})$

$t_{i}^{b} \quad$ berthing time of vessel $i\left(i \in \mathcal{V}, t_{i}^{b} \in \mathcal{T}\right)$

$\tilde{t}_{i}^{d} \quad$ actual departure time of vessel $i\left(i \in \mathcal{V}, \tilde{t}_{i}^{d} \in \mathcal{T}\right)$

$\theta_{i j}^{1} \quad$ binary variable, it equals 1 if vessel $j(j \in \mathcal{V})$ is located to the right-hand of vessel $i(i \in \mathcal{V})$, otherwise 0

$\theta_{i j}^{2} \quad$ binary variable, it equals 1 if vessel $j(j \in \mathcal{V})$ is located above vessel $i(i \in \mathcal{V})$, otherwise 0

$\eta_{\text {nit }}$ binary variable, it equals 1 if $n\left(n \in N_{i}\right)$ QCs serve vessel $i(i \in \mathcal{V})$ at time $t(t \in \mathcal{T})$, otherwise 0

\section{Auxiliary variables}

$\Delta x_{i} \quad$ the deviation of vessel $i(i \in \mathcal{V})$ between berth position and its desired position

$\Delta t_{i}^{d} \quad$ departure delay time of vessel $i(i \in \mathcal{V})$

$z \quad$ the quantity of carbon emission (tons)

Let $\mathbf{x}$ denote the vector of all decision variables and auxiliary variables, namely $\mathbf{x}:=\left(x_{i}, t_{i}^{b}, q_{i} / \hat{b}, \theta_{i j}^{1}, \theta_{i j}^{2}, \eta_{n i t}, \Delta x_{i}, \Delta t_{i}^{d}, i, j \in \mathrm{V}, n \in N_{i}, t \in \mathrm{T}:\right)$. As mentioned earlier, the BOIP model is to seek the optimal trade-off between service efficiency and operating cost saving for a given planning horizon. The two objectives in the BOIP model are as follows:

Objective 1:

$$
F_{1}(\mathbf{x})=\sum_{i \in \mathrm{V}:}\left(t_{i}^{b}-t_{i}^{a}\right)+\sum_{i \in \mathrm{V}:} \Delta t_{i}^{d}
$$

Objective 2:

$$
F_{2}(\mathbf{x}, z)=C^{F C}(\mathbf{x})+C^{C T}(z)
$$

where $C^{F C}(\mathbf{x})$ denotes the fundamental cost which is given by Eq.(5)

$$
C^{F C}(\mathbf{x})=c \times\left(\sum_{i \in \mathrm{V}: t \in \mathrm{T}} \sum_{n \in N_{i}} n \times \eta_{n i t}\right)
$$

Therefore, the BAQCAP is formulated as below:

[Model 1]

$$
\min _{\mathbf{x}, z} F(\mathbf{x}, z)=\left(F_{1}(\mathbf{x}), F_{2}(\mathbf{x}, z)\right)
$$

s.t.

$$
\begin{gathered}
x_{i}+l_{i} \leq L \quad \forall i \in \mathrm{V} \\
t_{i}^{b} \geq t_{i}^{a} \quad \forall i \in \mathrm{V} \\
x_{i}+l_{i} \leq x_{j}+M\left(1-\theta_{i j}^{1}\right) \quad \forall i, j \in \mathrm{V}
\end{gathered}
$$




$$
\begin{aligned}
& 9 / 8 \leq t_{j}^{b}+M\left(1-\theta_{i j}^{2}\right) \quad \forall i, j \in \mathrm{V} \\
& 1 \leq \theta_{i j}^{1}+\theta_{j i}^{1}+\theta_{i j}^{2}+\theta_{j i}^{2} \leq 2 \quad \forall i, j \in \mathrm{V}, i<j \\
& \Delta x_{i} \geq x_{i}-l_{i}^{b} \quad \forall i \in \mathrm{V} \\
& \Delta x_{i} \geq l_{i}^{b}-x_{i} \quad \forall i \in \mathrm{V} \\
& \Delta t_{i}^{d} \geq 1 / t_{i}-t_{i}^{d} \quad \forall i \in \mathrm{V} \\
& \Delta t_{i}^{d} \geq 0 \quad \forall i \in \mathrm{V} \\
& \frac{\left(1+\hat{\alpha} \cdot \Delta x_{i}\right) W_{i}}{\left(N_{i}^{\max }\right)^{20}} \leq q_{i} / b-t_{i}^{b} \leq \frac{\left(1+\hat{\alpha} \cdot \Delta x_{i}\right) W_{i}}{\left(N_{i}^{\min }\right)^{20}} \quad \forall i \in \mathrm{V} \\
& x_{i} \geq 0 ; t_{i}^{b}, q / 6 \in \mathrm{T} \quad \forall i \in \mathrm{V} \\
& \theta_{i j}^{1}, \theta_{i j}^{2} \in\{0,1\} \quad \forall i, j \in \mathrm{V} \\
& \sum_{t \in \mathrm{T}} \sum_{n \in N_{i}} n^{\%} \times \eta_{n i t} \geq\left(1+\hat{\alpha} \cdot \Delta x_{i}\right) W_{i} \quad \forall i \in \mathrm{V} \\
& \sum_{i \in \mathrm{V}} \sum_{n \in N_{i}} n \times \eta_{n i t} \leq Q \quad \forall t \in \mathrm{T} \\
& \sum_{n \in N_{i}} \eta_{n i t}=1 \quad \forall i \in \mathrm{V}, t \in\left[t_{i}^{b}, \ell_{i} / t^{\prime}-1\right] \\
& \eta_{n i t} \in\{0,1\} \quad \forall i \in \mathrm{V}, n \in N_{i}, t \in \mathrm{T} \\
& z=\mu \times\left(\sum_{i \in \mathrm{V}: t \in \mathrm{T}} \sum_{n \in N_{i}} n \times \eta_{n i t}\right) \times \alpha
\end{aligned}
$$

Eq. (6) is the objective function to Model 1, which minimizes the summation of berth waiting time and departure delay time, and minimizes the summation of fundamental cost and carbon emission tax as well. Constraints (7) ensure that each vessel $i$ can be berthed on the boundary of the wharf. Constraints (8) indicate that each vessel $i$ should berth after it arrives at the port. Constraints (9)-(11) enforce the non-overlapping conditions for vessels in the 2-dimensional berth-time plane (see Park and Kim (2003) for details), and $M$ is a sufficiently large positive constant. The berth deviation $\Delta x_{i}:=\left|l_{i}^{b}-x_{i}\right|$ and it can be equivalently determined by Constraints (12) and (13). The departure delay time of vessel $i(i \in \mathcal{V})$ denoted by $\Delta t_{i}^{d}$ which is equal to $\tilde{t}_{i}^{d}-t_{i}^{d}$ if $\tilde{t}_{i}^{d}>t_{i}^{d}$ and zero otherwise, it is given by Constraints (14) and (15). When vessel $i$ deviates $\Delta x_{i}$ units away from its desired position, the port operator has to arrange $\left(1+\hat{\alpha} \Delta x_{i}\right) W_{i}$ raw QC hours to serve the vessel. 
Obviously, the assignment of $N_{i}^{\text {min }}$ QCs to the vessel results in the longest time duration of the vessel mooring at berth, and conversely, the assignment of $N_{i}^{\max }$ QCs results in the shortest time duration. Therefore, the time duration of vessel $i$ mooring at berth for being served satisfies Constraints (16). Constraints (17) and (18) define the domains for integer decision variables and binary decision variables for berth allocation, respectively. The left side of constraints (19) is the productivity obtained from assigning $n$ QCs to vessel $i$ within a given interference exponent $\tilde{\alpha}(0<$ $\tilde{\alpha} \leq 1$ ); the right side of constraints (19) is the modified workload of the vessel for a given berth deviation factor $\hat{\alpha}(\hat{\alpha} \geq 0)$ and berth deviation $\Delta x_{i}$ between the required position $l_{i}^{b}$ and the actual position $x_{i}$ (Meisel and Bierwirth, 2009). Constraints (20) imply that the QCs serving vessel $i$ at any time cannot exceed the number of available QCs. Constraints (21) show that QCs are assigned to vessels during its turnaround time. Constraints (22) define the domains for binary decision variables for QC assignment. Eq. (23) gives the quantity of carbon emission.

It is noted that there are two differences between our model from the existing models developed by Meisel and Bierwirth (2009), Li et al. (2015), Iris et al. (2015, 2017), and He (2016). One difference is the form of models. As mentioned before, the models in Meisel and Bierwirth (2009), Li et al. (2015) and Iris et al. $(2015,2017)$ are all single-objective to minimize the total costs, and the model in He (2016) is bi-objective considering the departure delay of all vessels and the total handling energy consumption of all QCs. Second, in Section 2.1, we also point out the differences between energy consumption and carbon emissions in the objectives which differentiate our study from He (2016) and justifies the contributions of our study.

\section{Solution method}

Before we develop the solution method to solve Model 1 for the proposed BAQCAP with consideration of carbon emission taxation, we firstly explore the difficulties to solve the model. Firstly, Model 1 is a BIOP model, and neither solvers nor computer modeling language exists for it (Stidsen et al. 2014). Secondly, the BAQCAP is computationally challenging, as it is more complex than berth allocation problem (BAP) which is known to be strongly NP-hard (Lim, 1998; Xu and Lee, 2018). Thirdly, the objective function of Model 1 in Eq. (6) involves a piecewise linear cost function (i.e. Objective 2), which increases the hardness. These difficulties make the existing solution methods used to solve traditional BAQCAPs cannot be directly applied here. Therefore, it is a challenge for us to develop an efficient solution method to solve Model 1. In Section 5.1, we illustrate the procedures of 
balanced box method proposed by Boland et al. (2015) to solve general BIOPs, and then in Section 5.2, we develop a heuristic method to solve the optimization problems involved in the balanced box method.

\subsection{The balanced box method to solve the BOIP Models}

There are some popular scalarization techniques for solving BOIPs, such as the weighted sum method (Aneja and Nair, 1979), the perpendicular search method (Chalmet et al., 1986), the augmented weighted Tchebycheff method (Bowman, 1976; Steuer and Choo, 1983), and the $\epsilon-$ constraint method (Chankong and Haimes, 1983), etc. However, these methods each have their own weakness: the weighted sum method can only find all extreme supported nondominated points; the perpendicular search method may not be efficient because half of the optimization problems solved are infeasible and integer programming solvers tend to struggle when proving infeasibility; the augmented weighted Tchebycheff method may not be efficient because integer programming solves tend to toil when faced with min-max objectives; and the $\epsilon$-constraint method is not suited for obtaining high-quality approximate efficient frontiers quickly.

Recently, Boland et al. (2015) proposed the balanced box method to solve BIOPs, and stated that the method can remedy all weaknesses of those methods mentioned above and it outperforms them as well. Therefore, we adopt the balanced box method to solve Model 1. Its procedures can be briefly illustrated as below:

Balanced box method Obtain Pareto frontier to Model 1

Step 0: Initialize. Let $\epsilon>0$ be a small constant. Denote with $\mathcal{R}\left(F^{1}, F^{2}\right)$ the rectangle to be investigated where $F^{1}$ and $F^{2}$ represents the upper left and lower right corners of rectangle $\mathcal{R}$, respectively. Set $F^{1}=(0,+\infty)$ and $F^{2}=(+\infty, 0)$. Denote the set of rectangles by $\Re$ and set $\mathfrak{R}=\left\{\mathcal{R}\left(F^{1}, F^{2}\right)\right\}$. Denote the current set of nondominated points by $\mathcal{S}$ and set $\mathcal{S}=$ $\emptyset$.

Step 1: Update the points $F^{1}$ and $F^{2}$ in the initial rectangle $\mathcal{R}$. Solve the lexicographic optimization problems $F^{1}:=\operatorname{lexmin}\left\{F_{1}, F_{2}: F \in \mathcal{R}\right\}$ and $F^{2}:=\operatorname{lexmin}\left\{F_{2}, F_{1}: F \in \mathcal{R}\right\}$ to update $F^{1}$ and $F^{2}$, respectively. Then, $F^{1}$ and $F^{2}$ are the upper left and lower right points of Pareto frontier, respectively, and add them into set $\mathcal{S}$. Update set $\mathfrak{R}$.

Step 2: Halve rectangle. For each rectangle $\mathcal{R}\left(F^{1}, F^{2}\right) \in \Re$, horizontally halve it into two rectangles, 
denoted by $\mathcal{R}^{T}$ and $\mathcal{R}^{B} . \mathcal{R}^{T}:=\mathcal{R}\left(F^{1},\left(F_{1}^{2}, \frac{F_{2}^{1}+F_{2}^{2}}{2}\right)\right)$ and $\mathcal{R}^{B}:=\mathcal{R}\left(\left(F_{1}^{1}, \frac{F_{2}^{1}+F_{2}^{2}}{2}\right), F^{2}\right)$. Add $\mathcal{R}^{T}$ and $\mathcal{R}^{B}$ into set $\mathfrak{R}$ and remove the original rectangle $\mathcal{R}\left(F^{1}, F^{2}\right)$ from set $\mathfrak{R}$.

Step 3: Investigate rectangle $\mathcal{R}^{B}$. Solve the lexicographic optimization problem $\bar{F}^{1}:=$ $\operatorname{lexmin}\left\{F_{1}, F_{2}: F \in \mathcal{R}^{B}\right\}$ to obtain a nondominated point $\bar{F}^{1}$ in rectangle $\mathcal{R}^{B}$. If $\bar{F}^{1} \neq F^{2}$, then add $\bar{F}^{1}$ into set $\mathcal{S}$, and update $\mathcal{R}^{B}=\mathcal{R}\left(\bar{F}^{1}, F^{2}\right)$, and go to Step 4. Otherwise remove rectangle $\mathcal{R}^{B}$ from set $\Re$ and go to Step 5 .

Step 4: Modify rectangle $\mathcal{R}^{T}$. It is modified as $\mathcal{R}^{T}=\mathcal{R}\left(F^{1},\left(\bar{F}_{1}^{1}-\epsilon, \frac{F_{2}^{1}+F_{2}^{2}}{2}\right)\right)$.

Step 5: Investigate rectangle $\mathcal{R}^{T}$. Solve the lexicographic optimization problem $\bar{F}^{2}:=$ $\operatorname{lexmin}\left\{F_{2}, F_{1}: F \in \mathcal{R}^{T}\right\}$ to obtain a nondominated point $\bar{F}^{2}$ in rectangle $\mathcal{R}^{T}$. If $\bar{F}^{2} \neq F^{1}$, then add $\bar{F}^{2}$ into set $\mathcal{S}$, and update $\mathcal{R}^{T}=\mathcal{R}\left(F^{1}, \bar{F}^{2}\right)$. Otherwise remove rectangle $\mathcal{R}^{T}$ from set $\mathfrak{R}$. Go to Step 6.

Step 6: Stop criterion check. If set $\mathfrak{R}$ is empty, then stop and output the current set $\mathcal{S}$. Otherwise go back to Step 2.

The lexicographic optimization problem $\operatorname{lexmin}\left\{F_{1}, F_{2}: F \in \mathcal{R}^{B}\right\}$ is defined as follows:

$$
\bar{F}_{1}^{1}:=\min \left\{F_{1} \mid(7)-(23) \text {, and } F \in \mathcal{R}^{B}\right\}
$$

followed by

$$
\bar{F}_{2}^{1}:=\min \left\{F_{2} \mid(7)-(23), F \in \mathcal{R}^{B}, \text { and } F_{1} \leq \bar{F}_{1}^{1}\right\}
$$

Similarly, lexmin $\left\{F_{2}, F_{1}: F \in \mathcal{R}^{T}\right\}$ is defined as follows:

$$
\bar{F}_{2}^{2}:=\min \left\{F_{2} \mid(7)-(23) \text {, and } F \in \mathcal{R}^{T}\right\}
$$

followed by

$$
\bar{F}_{1}^{2}:=\min \left\{F_{1} \mid(7)-(23), F \in \mathcal{R}^{T} \text {, and } F_{2} \leq \bar{F}_{2}^{2}\right\}
$$

It can be seen that the lexicographic optimization problem $\operatorname{lexmin}\left\{F_{1}, F_{2}: F \in \mathcal{R}^{B}\right\}$ corresponds to the preference of service efficiency, while the lexicographic optimization problem $\operatorname{lexmin}\left\{F_{2}, F_{1}: F \in \mathcal{R}^{T}\right\}$ corresponds to the preference of cost-saving.

\subsection{The heuristic method to solve the lexicographic optimization problems}

The procedures of the balanced box method described above require to iteratively solve a number of lexicographic optimization problems in order to obtain the nondominated points of Model 1. Each lexicographic optimization problem contains two single-objective optimization models for BAQCAP. It is noted that CPLEX is quite cumbersome and time-consuming to solve the single-objective 
optimization models formulated for the traditional BAQCAP in the existing literature. As reported by Meisel and Bierwirth (2009), Li et al. (2015), Iris et al. (2015), Vacca et al. (2015), etc., CPLEX took several hours even for small-scale instances. As for the solution algorithms of the literatures reviewed in Section 2.1, they cannot be directly used or they are inappropriate to solve our single-objective optimization models, because our single-objective optimization models are different with their models. Recently, Xu and Lee (2018) propose a method for the BAP and extend this method for BAQCAP, but their method cannot be applied here because it requires invariant QC assignment, while ours is variant (the decision variable $\eta_{\text {nit }}$ reflects the variation). Therefore, we need to design a new and appropriate heuristic method to quickly solve the optimization models in the lexicographic optimization problems. Before we construct a heuristic method, let us firstly explore the properties of Model 1 . We find that it has the following properties:

1) Model 1 can be divided into two phases: Phase 1 is berth plan which determines berthing position and berthing time satisfying constraints (7)-(18); Phase 2 is QC assignment which determines the number of QCs to vessels satisfying constraints (19)-(22) (Eq.(23) just calculates the quantity of carbon emission once the QC assignment is determined in Phase 2). Obviously, QC assignment in Phase 2 can be obtained only when the berth plan in Phase 1 is known. That is, the output to Phase 1 is an input to Phase 2.

2) It's apparent that the berth waiting time of vessel $i$ linearly increases with the value of berthing time $t_{i}^{b}$. When vessel $i$ deviates $\Delta x_{i}$ units away from its desired position, the workload increases from $W_{i}$ to $\left(1+\hat{\alpha} \Delta x_{i}\right) W_{i}$, which shows that the workload also linearly increases with the value of $\Delta x_{i}$.

3) Given the adjusted workload of vessel $i$ with a deviation of $\Delta x_{i}$, the turnaround time of the vessel moored at berth, denoted by $t_{i}\left(t_{i}:=\tilde{t}_{i}^{d}-t_{i}^{b}\right)$, decreases with the number of QCs assigned to the vessel.

4) As $n^{1-\widetilde{\alpha}}$ increases with respect to $n$, the number of QC hours needed to finish the workload of vessel $i$ with a given deviation of $\Delta x_{i}$ increases with the number of QCs assigned to the vessel.

5) It's apparent that QCs cannot be assigned to vessel $i$ before the vessel moors at berth neither after the vessel departs, which indicates that the decision variables of QC assignment, $\eta_{\text {nit }}$, should be set as $\eta_{\text {nit }}=0$ for $t<t_{i}^{b}$ and $t \geq \tilde{t}_{i}^{d}$. 
The framework of the heuristic to obtain a nondominated point $\bar{F}^{1}$ in rectangle $\mathcal{R}^{B}$ by solving the lexicographic optimization problem lexmin $\left\{F_{1}, F_{2}: F \in \mathcal{R}^{B}\right\}$, is termed as Heuristic $\bar{F}^{1}$ for sake of presentation, and is composed of three steps. The first step obtains an initial nondominated point, the second step iteratively improves the initial nondominated point until a termination condition is satisfied, and the third step outputs the final nondominated point $\bar{F}^{1}$. In order to obtain an initial nondominated point in step 1, three sub-steps are proceeded: 1) sort vessels by their initial priorities, 2) insert vessel $i$ into the space-time diagram and select a best berthing time and berthing position for vessel $i$, and determine the optimal departure time and QC assignment for vessel $i, 3$ ) obtain the initial nondominated point once all vessels are inserted. Then, step 2 repeats the same steps to improve the initial nondominated point until a termination condition is fulfilled. The sub-algorithm to each step in Heuristic $\bar{F}^{1}$ is stated in the following sections.

\subsubsection{Priority rule of vessels}

Some studies have proposed different rules to assign priorities to vessels for berthing. Guan and Cheung (2004) utilize the First-Come-First-Served (FCFS) principle. Meisel and Bierwirth (2009) initialize priorities of vessels by FCFS principle and then adjust the priorities by squeaky wheel optimization and tabu search. Lee et al. (2010) adopt FCFS to assign initial priority and produced new priorities by swapping two adjacent vessels. Li et al. (2015) develop an initial priority rule based on pessimistic scenarios and constructed a new priority list according to vessel penalty costs. However, as stated by Li et al. (2015), a high-quality berthing plan cannot be obtained according to FCFS. The priority rule proposed in $\mathrm{Li}$ et al. (2015) is inappropriate for our problem because we have no penalty cost for vessels here. Therefore, we need to propose a priority rule to insert vessels into the space-time diagram, which is the first contribution of our solution algorithm.

It's found that vessels have different sensitivities to their preferred operational productivities. For instance, some late-arriving and short-mooring vessels require completing all operations with high operational productivities so that they can depart on time. If the FCFS principle is adopted, these vessels maybe cannot be assigned to sufficient QCs because of restricted QC resource, resulting in departure delays. Thus, it's rationale to preferentially provide operational service for these vessels.

Here, an initial priority rule is based on expected turnaround time moored at berth, which is used to evaluate the sensitivities of vessels for their preferred operational productivity. In this initial priority rule, vessel $i$ is moored at its desired position $l_{i}^{b}$ and operated at its expected time window 
$\left[t_{i}^{a}, t_{i}^{d}\right]$. Therefore, the operational productivity required by vessel $i$ is given by $p_{i}:=\frac{w_{i}}{t_{i}^{d}-t_{i}^{a}}$. Then, sort the vessels by descending order of $p_{i}$ to obtain the initial priority order of vessels. Then vessels are inserted by their priorities into the space-time diagram, and their berth-QC plans can be obtained.

In the iterative procedures, the priorities of all vessels for a new iteration can be evaluated by the operational productivity based on berth-QC plan in last iteration. When an iteration is finished, the berth-QC plans for all vessels are updated, then the operational productivity required by vessel $i$ can be updated as $p_{i}=\frac{\left(1+\widehat{\alpha} \Delta x_{i}\right) W_{i}}{\tilde{t}_{i}^{d}-t_{i}^{b}}$. Then, sort the vessels by descending order of $p_{i}$ to obtain the new priority order of vessels for next iteration. The iteration procedure terminates when a repeated priority list is generated or the iteration number threshold is met.

\subsubsection{Heuristic for berthing time and berthing position}

With a given priority order of all vessels produced in Section 5.2.1, the vessels are inserted into the space-time diagram one by one according to their priorities. Based on Property 1, the berth plan problem in Phase 1 is solved before the QC assignment problem in Phase 2. Therefore, we firstly construct a heuristic to obtain the berthing time and berthing position for a vessel to be inserted in this section, and then construct a heuristic to departure time and assign QCs to this vessel in next section.

For the berth plan problem in Phase 1, Property 2 tells that each vessel prefers to berth at the time as close as possible to its expect arrival time, and berth at the position as close as possible to its desired position, so as to minimize its berth waiting time and the additional workload caused by position deviation, respectively. Thus, the perfect berthing time and berthing position for vessel $i(i \in \mathcal{V})$ are $t_{i}^{b}=t_{i}^{a}$ and $x_{i}=l_{i}^{b}$, respectively. Li et al. (2015) insert vessels based on its preferred position. However, the perfect berth plans for some vessels may be infeasible because of overlapping of either turnaround time or berthing space. Meisel and Bierwirth (2009) look for a good berth plan for vessel $i(i \in \mathcal{V})$ by probing each time and position unit close around $t_{i}^{a}$ and $l_{i}^{b}$ on the space-time diagram. However, such a probing method is inefficient. Enlightened by Guan and Cheung (2005), we can propose a convenient method to quickly find a good berthing time and berthing position for a vessel to be inserted, see the following proposition:

Proposition 2. Given a priority order of all vessels denoted by $\left\{o_{1}, \ldots, o_{i}, \ldots, o_{V}\right\}$, where $o_{i}$ represent the order of vessel $i$, there is an optimal berth plan in which each vessel $i(i \in \mathcal{V})$ to be inserted moors at its expected arrival time or at the time immediately after another vessel's departure (namely 
$t_{i}^{b}=t_{i}^{a}$ or $t_{i}^{b}=\tilde{t}_{j}^{d}$ for some $j \in \overline{\mathcal{V}}$ ), and moors at its desired position or at the position at immediately right of another vessel (namely $x_{i}=l_{i}^{b}$ or $x_{i}=x_{j}+l_{j}$ for some $j \in \overline{\mathcal{V}}$ ), where $\overline{\mathcal{V}}$ denotes the set of vessels which have been inserted and $|\overline{\mathcal{V}}|=o_{i}-1$.

Proof: The proof is similar to the proofs of Properties 1 3 in Kim and Moon (2003) when we replace the first term in their objective function by the berth waiting time shown in Eq. (3).

Proposition 2 shows that we can choose a good berthing time and berthing position for vessel $i$ from three sets of candidate points on the space-time diagram: $\left\{\left(l_{i}^{b}, t_{i}^{a}\right),\left(l_{i}^{b}, \tilde{t}_{j}^{d}\right)\right\},\left\{\left(x_{j}+l_{j}, t_{i}^{a}\right)\right\}$, and $\left\{\left(x_{j}+l_{j}, \tilde{t}_{j}^{d}\right)\right\}$. The first set prefers to insert vessel $i$ at its desired position but maybe with berth waiting, the second set prefers to moor vessel $i$ at the berth on its scheduled time but with position deviation, and the third set is with both. Obviously, the points contained in the former two sets are better than that of the third set. As position deviations of vessels result in additional workload, consequently, more turnaround time of vessels may need and result in departure delay. Additionally, more QC-hours are required which indicates more fundamental cost and carbon emission taxation. Hence, it is reasonable to regard that the first set is prior to the second set. Then, we can assign priorities to all candidate points according the rule, that is a point with good berthing position is prior to a point with good berthing time, and named it as berth plan rule. We follow this rule to search a good point for a vessel to be inserted, which is the second contribution of our solution algorithm.

It is noted that we need to check the feasibility of the candidate points. Recall that vessels are represented as rectangles in the space-time diagram and the width of rectangles represent the turnaround time of vessels, which equal to $\tilde{t}_{i}^{d}-t_{i}^{b}$. When a point is selected for vessel $i$, the berthing time $t_{i}^{b}$ and berthing position $x_{i}$ of vessel $i$ is given as the coordinate of the point, but the actual departure time $\tilde{t}_{i}^{d}$ is unknown yet, which means that the width of rectangle for vessel $i$ is unknown. However, there is no feasibility check in Guan and Cheung (2005) and Lee et al. (2010), because the turnaround time in their studies is known. Meisel and Bierwirth (2009) check the feasibility of a selected point for vessel $i$, but the QC assignment for vessel $i$ requires to obtain simultaneously, which means that an optimization problem to assign QCs has to be solved. Li et al. (2015) cluster the vessels with spatiotemporal conflicts and formulate a resource assignment model for clustered vessels to resolve the conflicts by a branch-and-bound method. The methods of Meisel and Bierwirth (2009) and Li et al. (2015) are both cumbersome. Therefore, we need to propose a 
simple method to quickly check the feasibility here. Property 3 shows that the latest departure time $\tilde{t}_{i}^{d}$ for vessel $i$ is $\tilde{t}_{i}^{d}=\left\lceil t_{i}^{b}+\frac{\left(1+\widehat{\alpha} \Delta x_{i}\right) W_{i}}{\left(N_{i}^{\text {min }}\right)^{\tilde{\alpha}}}\right\rceil$. Then, we can check the feasibility of the selected point for vessel $i$ by this way: if the rectangle with largest width for vessel $i$ does not overlap with other rectangles for vessel $j \in \overline{\mathcal{V}}$, i.e. constraints (9)-(11) are fulfilled, then the selected point for vessel $i$ is definitely feasible, which is the third contribution of our solution algorithm.

Now, we propose the heuristic to select a good and feasible point for vessel $i(i \in \mathcal{V})$ which is to be inserted based on the above analysis, and describe it as below:

Sub-algorithm 1 Select a best and feasible point to insert vessel $i$

Input: Priority order of all vessels $\left\{o_{1}, \ldots, o_{i}, \ldots, o_{V}\right\}$, set of inserted vessels $j \in \overline{\mathcal{V}}$, sets of candidate points $\mathbb{S}:=\left\{\left(l_{i}^{b}, t_{i}^{a}\right),\left(l_{i}^{b}, \tilde{t}_{j}^{d}\right),\left(x_{j}+l_{j}, t_{i}^{a}\right),\left(x_{j}+l_{j}, \tilde{t}_{j}^{d}\right)\right\}$ indexed by $k(k \in \mathbb{S}), \quad\left(N_{i}^{\min }, N_{i}^{\max }, W_{i}\right)$ for vessel $i$.

Output: A best berthing time and berthing position of vessel $i(i \in \mathcal{V})$ to be inserted

Step 1: for all $k(k \in \mathbb{S})$ do

Follow Berth plan rule. Rank all candidate points $k(k \in \mathbb{S})$ by their priorities.

\section{end for}

Step 2: Select a good and feasible point. Let $k=1$.

Step 2.1: Obtain the berthing time $t_{i}^{b}$ and berthing position $x_{i}$ for vessel $i$

Step 2.2: Obtain the latest departure time $\tilde{t}_{i}^{d}$ for vessel $i$ by setting $\tilde{t}_{i}^{d}=\left\lceil t_{i}^{b}+\frac{\left(1+\widehat{\alpha} \Delta x_{i}\right) W_{i}}{\left(N_{i}^{\min }\right)^{\tilde{\alpha}}}\right\rceil$

Step 2.3: Check non-overlapping conditions of vessel $i$ with vessels $j(j \in \overline{\mathcal{V}})$

if non-overlapping conditions (9)-(11) satisfy, then

result $=$ point $k$

else

Update $k=k+1$. Go back to Step 2.1 .

end if

return result.

\subsubsection{Heuristics to departure time and QC assignment for vessels}

It can be seen that sub-algorithm 1 described above only gives the berthing time $t_{i}^{b}$ and berthing position $x_{i}$ for vessel $i$, now we determine the actual departure time $\tilde{t}_{i}^{d}$ and QC assignment $\eta_{n i t}$ 
for vessel $i$ in this section. Recall that the lexicographic optimization problems $\operatorname{lexmin}\left\{F_{1}, F_{2}: F \in\right.$ $\left.\mathcal{R}^{B}\right\}$ and lexmin $\left\{F_{2}, F_{1}: F \in \mathcal{R}^{T}\right\}$ correspond to the preference of service efficiency and cost-saving, respectively, and yield different results of $\tilde{t}_{i}^{d}$ and $\eta_{n i t}$. Consequently, we need to propose the sub-lexicographic optimization problems for vessel $i$ for the two different preferences, denoted by $\operatorname{lexmin}\left\{f_{i 1}, f_{i 2}\right\}$ and $\operatorname{lexmin}\left\{f_{i 2}, f_{i 1}\right\}$, respectively. For the sake of presentation, we first define the objectives in the sub-lexicographic optimization problems for vessel $i$. Let

$$
\text { Objective 1: } \quad f_{i 1}\left(q_{i} / b, \Delta t_{i}^{d}\right)=t_{i}^{b}-t_{i}^{a}+\Delta t_{i}^{d}
$$

$$
\text { Objective 2: } \quad f_{i 2}\left(\eta_{n i t}, z_{i}\right)=C_{i}^{F C}\left(\eta_{n i t}\right)+C_{i}^{C T}\left(z_{i}\right)
$$

where $C_{i}^{F C}\left(\eta_{n i t}\right)$ denotes the fundamental cost of vessel $i$ which is given by Eq.(26)

$$
C_{i}^{F C}\left(\eta_{n i t}\right)=c \times \sum_{t \in \mathrm{T}} \sum_{n \in N_{i}} n \times \eta_{n i t}
$$

$C_{i}^{C T}\left(z_{i}\right)$ denotes the carbon emission taxation of vessel $i$ where $z_{i}$ is the amount of carbon emission of QCs assigned to vessel $i$. Recall that vessels are inserted one by one according to their priorities, thus the berth-QC plans and carbon emission of inserted vessels $j \in \overline{\mathcal{V}}$ are known. The total carbon emission of vessels $j \in \overline{\mathcal{V}}$ can be calculated by $\sum_{j \in \overline{\mathcal{V}}} z_{j}$, and we let $s_{0}$ represent the number of the segment it locates in, which indicates that $\sum_{j \in \overline{\mathcal{V}}} z_{j} \in\left[\Gamma_{S_{0}-1}, \Gamma_{S_{0}}\right)$. If $\sum_{j \in \overline{\mathcal{V}} \cup\{i\}} z_{j} \in$ $\left[\Gamma_{s-1}, \Gamma_{s}\right)$, then $C_{i}^{C T}\left(z_{i}\right)$ is given by

$$
C_{i}^{C T}\left(z_{i}\right)=r_{s} \times \sum_{j \in \mathbb{V} \cup\{i\}} z_{j}+b_{s}-r_{s_{0}} \times \sum_{j \in \mathbb{V}} z_{j}-b_{s_{0}}
$$

The constraints in sub-lexicographic optimization problems for vessel $i$ are as follows:

$$
\begin{aligned}
& \Delta t_{i}^{d} \geq 9 t_{i}^{d}-t_{i}^{d} \\
& \Delta t_{i}^{d} \geq 0 \\
& \frac{\left(1+\hat{\alpha} \cdot \Delta x_{i}\right) W_{i}}{\left(N_{i}^{\max }\right)^{\alpha_{0}}} \leq q_{i} / \bar{b}-t_{i}^{b} \leq \frac{\left(1+\hat{\alpha} \cdot \Delta x_{i}\right) W_{i}}{\left(N_{i}^{\min }\right)^{20}} \\
& 9 / 6 \in T \\
& \sum_{t \in \mathrm{T}} \sum_{n \in N_{i}} n^{\%} \times \eta_{n i t} \geq\left(1+\hat{\alpha} \cdot \Delta x_{i}\right) W_{i} \\
& \sum_{n \in N_{i}} n \times \eta_{n i t} \leq Q_{i t} \quad \forall t \in\left[t_{i}^{b}, q / h_{i}-1\right] \\
& \sum_{n \in N_{i}} \eta_{n i t}=1 \quad \forall t \in\left[t_{i}^{b}, q_{i}^{q / 6}-1\right]
\end{aligned}
$$




$$
\begin{gathered}
\eta_{n i t} \in\{0,1\} \quad \forall n \in N_{i}, t \in \mathrm{T} \\
z_{i}=\mu \times\left(\sum_{t \in \mathrm{T}} \sum_{n \in N_{i}} n \times \eta_{n i t}\right) \times \alpha
\end{gathered}
$$

where $Q_{i t}$ denotes the number of available QCs for vessel $i$ at time $t$. Recall again that the berth-QC plans of vessels $j \in \overline{\mathcal{V}}$ have been determined already, so when vessel $i$ is to be inserted, $Q_{i t}$ is known and equals to $Q-\sum_{j \in \overline{\mathcal{V}}} \sum_{n \in N_{j}} n \times \eta_{n j t}$.

It is noted that a point $F:=\left(F_{1}, F_{2}\right)$ is given by $F_{1}=\sum_{i=1}^{i=V} f_{i 1}$ and $F_{2}=\sum_{i=1}^{i=V} f_{i 2}$, so the constraint $F \in \mathcal{R}^{B}$ in the lexicographic optimization problem $\operatorname{lexmin}\left\{F_{1}, F_{2}: F \in \mathcal{R}^{B}\right\}$ can be replaced by $F_{1}^{1} \leq \sum_{i=1}^{i=V} f_{i 1} \leq F_{1}^{2}$ and $F_{2}^{2} \leq \sum_{i=1}^{i=V} f_{i 2} \leq \frac{F_{2}^{1}+F_{2}^{2}}{2}, \mathcal{R}^{B}=\mathcal{R}\left(\left(F_{1}^{1}, \frac{F_{2}^{1}+F_{2}^{2}}{2}\right), F^{2}\right)$, where $F^{1}:=\left(F_{1}^{1}, F_{2}^{1}\right)$ and $F^{2}:=\left(F_{1}^{2}, F_{2}^{2}\right)$ are upper left and lower right corners of rectangle $\mathcal{R}$, and the constraint $F_{1} \leq \bar{F}_{1}^{1}$ can be replaced by $\sum_{i=1}^{i=V} f_{i 1} \leq \bar{F}_{1}^{1}$. Therefore, besides the constraints (28)-(36), additional constraints have to be included in $\operatorname{lexmin}\left\{f_{i 1}, f_{i 2}\right\}$ in order to guarantee the constraints $F \in \mathcal{R}^{B}$ and $F_{1} \leq \bar{F}_{1}^{1}$ in $\operatorname{lexmin}\left\{F_{1}, F_{2}: F \in \mathcal{R}^{B}\right\}$ be satisfied. Recall that $F^{1}:=\operatorname{lexmin}\left\{F_{1}, F_{2}: F \in\right.$ $\mathcal{R}\}$, then $F_{1}^{1}=\sum_{i=1}^{i=V} f_{i 1}^{1^{*}}$ and $F_{2}^{1}=\sum_{i=1}^{i=V} f_{i 2}^{1^{*}}$, where $f_{i 1}^{1^{*}}$ and $f_{i 2}^{1^{*}}$ are optimal objective function value to $f_{i 1}$ and $f_{i 2}$ in $\operatorname{lexmin}\left\{F_{1}, F_{2}: F \in \mathcal{R}\right\}$, respectively. Also, $F^{2}:=\operatorname{lexmin}\left\{F_{2}, F_{1}: F \in \mathcal{R}\right\}$, then $F_{2}^{2}=\sum_{i=1}^{i=V} f_{i 2}^{2^{*}}$ and $F_{1}^{2}=\sum_{i=1}^{i=V} f_{i 1}^{2^{*}}$, where $f_{i 2}^{2^{*}}$ and $f_{i 1}^{2^{*}}$ are optimal objective function value to $f_{i 2}$ and $f_{i 1}$ in $\operatorname{lexmin}\left\{F_{2}, F_{1}: F \in \mathcal{R}\right\}$, respectively. Therefore, the constraints $F \in \mathcal{R}^{B}$ in $\operatorname{lexmin}\left\{F_{1}, F_{2}: F \in \mathcal{R}^{B}\right\}$ can be satisfied by adding the following constraints in $\operatorname{lexmin}\left\{f_{i 1}, f_{i 2}\right\}$ :

$$
\begin{gathered}
f_{i 1}^{1^{*}} \leq f_{i 1} \leq f_{i 1}^{2^{*}} \\
f_{i 2}^{2^{*}} \leq f_{i 2} \leq \frac{f_{i 2}^{1^{*}}+f_{i 2}^{2^{*}}}{2}
\end{gathered}
$$

It is noted that according to the definition of $\operatorname{lexmin}\left\{F_{1}, F_{2}: F \in \mathcal{R}^{B}\right\}$ and $\operatorname{lexmin}\left\{F_{2}, F_{1}: F \in \mathcal{R}\right\}$, we have $f_{i 1}^{1^{*}} \leq f_{i 1}^{2^{*}}$ and $f_{i 2}^{2^{*}} \leq f_{i 2}^{1^{*}}$, respectively. Therefore, constraints (37) and (38) both hold. $F_{1} \leq \bar{F}_{1}^{1}$ in $\operatorname{lexmin}\left\{F_{1}, F_{2}: F \in \mathcal{R}^{B}\right\}$ can be guaranteed by adding the following constraint in $\operatorname{lexmin}\left\{f_{i 1}, f_{i 2}\right\}$ :

$$
f_{i 1} \leq \bar{f}_{i 1}^{1^{*}}
$$

where $\bar{f}_{i 1}^{1^{*}}$ is the optimal objective function value of $f_{i 1}$ in $\operatorname{lexmin}\left\{f_{i 1}, f_{i 2}\right\}$.

Therefore, the sub-lexicographic optimization problem $\operatorname{lexmin}\left\{f_{i 1}, f_{i 2}\right\}$ for vessel $i$ is finalized 
as follows:

$$
\bar{f}_{i 1}^{1^{*}}:=\min \left\{f_{i 1} \mid(28)-(38)\right\}
$$

followed by

$$
\bar{f}_{i 2}^{1^{*}}:=\min \left\{f_{i 2} \mid(28)-(39)\right\}
$$

As $\bar{f}_{i 1}^{1^{*}}$ and $\bar{f}_{i 2}^{1^{*}}$ both contain constraint (38) which involves a piecewise function $C_{i}^{C T}\left(z_{i}\right)$ in Eq.(27), the optimization solvers, such as CPLEX, etc., cannot be used to solve them directly. Enlightened by Croxton et al. (2003), the piecewise objective function $C_{i}^{C T}\left(z_{i}\right)$ can be linearized by introducing auxiliary variables. Let $\lambda_{s}$ be binary variable for each segment $s(s=1, \ldots, N)$, and define $z_{S}^{\prime}$ as a variable to represent the amount of carbon emission in segment $s$. Then, define $\tilde{C}_{i}^{C T}\left(z_{s}^{\prime}, \lambda_{s}\right):=\sum_{s=1}^{s=N}\left(b_{s} \lambda_{s}+z_{S}^{\prime} r_{s}\right)-r_{s_{0}} \times \sum_{j \in \overline{\mathcal{V}}} z_{j}-b_{s_{0}}$ as the carbon emission tax function for vessel $i$, thus, the objective function $f_{i 2}$ in (25) can be replaced by $\tilde{f}_{i 2}:=C_{i}^{F C}\left(\eta_{n i t}\right)+\tilde{C}_{i}^{C T}\left(z_{s}^{\prime}, \lambda_{s}\right)$. And the following constraints hold:

$$
\begin{gathered}
\sum_{s=1}^{N} z_{s}^{\prime}=\sum_{j \in \mathbb{V} \cup\{i\}} z_{j} \\
\sum_{s=1}^{N} \lambda_{s}=1 \\
\Gamma_{s-1} \lambda_{s} \leq z_{s}^{\prime} \leq \Gamma_{s} \lambda_{s} \quad \forall s \in\{1, \ldots, N\} \\
\lambda_{s} \in\{0,1\} \quad \forall s \in\{1, \ldots, N\} \\
z_{s}^{\prime} \geq 0 \quad \forall s \in\{1, \ldots, N\}
\end{gathered}
$$

It's straightforward that $\bar{f}_{i 1}^{1^{*}}$ defined in Eq. (40) is equivalent to the following problem (47):

$$
\tilde{\tilde{f}}_{i 1}^{1^{*}}:=\min \left\{f_{i 1} \mid(28)-(37),(42)-(46), \text { and } \tilde{f}_{i 2}^{2^{*}} \leq \tilde{f}_{i 2} \leq \frac{\tilde{f}_{i 2}^{*}+\tilde{f}_{i 2}^{*}}{2}\right\}
$$

and $\bar{f}_{i 2}^{1^{*}}$ defined in Eq.(41) is equivalent to the following problem (48):

$$
\overline{\tilde{f}}_{i 2}^{1^{*}}:=\min \left\{\tilde{f}_{i 2} \mid(28)-(37),(42)-(46), f_{i 1} \leq \overline{\tilde{f}}_{i 1}^{1^{*}}, \text { and } \tilde{f}_{i 2}^{2^{*}} \leq \tilde{f}_{i 2} \leq \frac{\tilde{f}_{i 2}^{*}+\tilde{f}_{i 2}^{2^{*}}}{2}\right\}
$$

Then, $\overline{\tilde{f}}_{i 1}^{1^{*}}$ in Eq.(47) and $\overline{\tilde{f}}_{i 2}^{1^{*}}$ in Eq.(48) can be obtained by CPLEX within a quite short time as they are small-scale problems. Particularly, the decision variables $\eta_{\text {nit }}$ for $t<t_{i}^{b}$ and $t \geq\left\lceil t_{i}^{b}+\right.$ $\frac{\left(1+\widehat{\alpha} \Delta x_{i}\right) W_{i}}{\left(N_{i}^{\min }\right)^{\tilde{\alpha}}} \mid$ can be preprocessed by setting $\eta_{\text {nit }}=0$ based on Property 5 before solving these problems. This will furtherly reduce the computation time. As for the sub-lexicographic optimization 
problem lexmin $\left\{f_{i 2}, f_{i 1}\right\}$, it is defined and solved by the $\operatorname{similar}$ way of $\operatorname{lexmin}\left\{f_{i 1}, f_{i 2}\right\}$.

\subsubsection{Heuristic to search for nondominated points in rectangles}

Based on the description and analysis above, we can describe Heuristic ${ }^{\bar{F}^{1}}$ to search for a nondominated point in rectangle $\mathcal{R}^{B}$ as below:

Heuristic $\overline{\boldsymbol{F}}^{1}$ Obtain a nondominated point $\bar{F}^{1}$ in rectangle $\mathcal{R}^{B}$

Input: Data of all vessels, i.e. $\left(N_{i}^{\min }, N_{i}^{\max }, W_{i}\right)$ for vessel $i$. Rectangles $\mathcal{R}^{B}$

Define Num as the number of iterations for vessel's priority and $n$ as the index of iteration

Output: A nondominated point $\bar{F}^{1}=\left(\bar{F}_{1}^{1}, \bar{F}_{2}^{1}\right)$ in $\mathcal{R}^{B}$

Step 1: Initialize.

Step 1.1 Obtain the initial priority of all vessels (heuristic in Sec. 5.2.1). Let $n=0$.

Step 1.2: for all vessels $i \in \mathcal{V}$ with initial priority order do

Select a best and feasible point for vessel $i$ (sub-algorithm 1 in Sec. 5.2.2)

Solve the optimization problem $f_{i 1}$ defined in Eq. (47) to obtain $\overline{\tilde{f}}_{i 1}^{1^{*}}$ by CPLEX

Solve the optimization problem $\tilde{f}_{i 2}$ defined in Eq. (48) to obtain $\overline{\tilde{f}}_{i 2}^{1^{*}}$ by CPLEX

end for

Step 1.3: Record $\bar{F}_{1}^{10}:=\sum_{i=1}^{i=V} \overline{\tilde{f}}_{i 1}^{1^{*}}$, and $\bar{F}_{2}^{10}:=\sum_{i=1}^{i=V} \overline{\tilde{f}}_{i 2}^{1^{*}}$

Step 2: Iteration.

for all iteration $1 \leq n \leq N u m$ do

Step 2.1 Update priorities of all vessels (heuristic in Sec. 5.2.1).

Step 2.2 for all vessels $i \in \mathcal{V}$ with updated priority order do

Repeat the procedures of selection and solving in Step 1.2

end for

Step 2.3 Record $\bar{F}_{1}^{1 n}:=\sum_{i=1}^{i=V} \overline{\tilde{f}}_{i 1}^{1^{*}}$, and $\bar{F}_{2}^{1 n}:=\sum_{i=1}^{i=V} \overline{\tilde{f}}_{i 2}^{1^{*}}$

end for

Step 3: Obtain $\bar{F}^{1}$.

Step 3.1 Let $n^{*} \in \underset{n \in\{0,1, \ldots, \text { Num }\}}{\operatorname{argmin}}\left\{\bar{F}_{1}^{1 n}\right\}$, and let $\tilde{n}^{*} \in \underset{n^{*} \in\{0,1, \ldots, \text { Num }\}}{\operatorname{argmin}}\left\{\bar{F}_{2}^{1 n^{*}}\right\}$

Step 3.2 Let $\bar{F}_{1}^{1}:=\bar{F}_{1}^{1 n^{*}}$ and $\bar{F}_{2}^{1}:=\bar{F}_{2}^{1 \tilde{n}^{*}}$

Step 3.3 Obtain $\bar{F}^{1}=\left(\bar{F}_{1}^{1}, \bar{F}_{2}^{1}\right)$ 
The heuristic to search for a nondominated point $\bar{F}^{2}$ in rectangle $\mathcal{R}^{T}$ (termed as Heuristic $\bar{F}^{2}$ ) is similar to Heuristic $\bar{F}^{1}$. Once all rectangles $\mathcal{R}^{B}$ and $\mathcal{R}^{T}$ in set $\Re$ are investigated, then all nondominated points to Model 1 are found.

\section{Computational investigations}

In this section, we conduct extensive numerical experiments to validate the effectiveness of the proposed model and the efficiency of the proposed solution algorithm. This section consists of two parts: one is performance analysis and the other is model analysis. Recall that the balanced box method is used to find all nondominated solutions of Model 1 in this paper, in which each nondominated solution is obtained by the proposed Heuristic $\bar{F}^{1}$ (or Heuristic $\bar{F}^{2}$ ), and the productivity rule is used to determine the priority of vessels in Heuristic $\bar{F}^{1}$ (or Heuristic $\bar{F}^{2}$ ). Therefore, in the performance analysis, we discuss the efficiency of the proposed solution method in terms of the computational time and solution quality from two aspects: 1) we compare Heuristic $\bar{F}^{\bar{F}^{1}}$ with CPLEX to show the efficiency of Heuristic $\bar{F}^{1}$ on solving a lexicographic optimization problem; and 2) we compare the productivity rule and FCFS rule to show the effectiveness of productivity rule on the initial solution of a lexicographic optimization problem. In the model analysis, we firstly explore the economic analysis of operating costs. Then we investigate the environmental analysis of carbon emission produced by QCs. All experiments are programmed using the programming language Lua and running on a PC with Intel (R) Core TM2 T9600@2.8 GHz processor and 4.0 GB of RAM. The optimization problems $f_{i 1}$ and $\tilde{f}_{i 2}$ are solved by CPLEX 12.6.

The method used to generate test instances is similar to that used in Iris et al. (2015), Li et al. (2015) and Meisel and Bierwirth (2009). The length of the quay is set at 100 units, and each unit is 10 $\mathrm{m}(L=1000 \mathrm{~m})$. The number of QCs available is set to 10 and the time unit is set to 1 hour. The vessels are distinguished into three classes: Feeder, Medium, and Jumbo, with different technical specifications (see Table 1). The data for these vessels, such as their length and workloads, are generated randomly as integer values with a uniform distribution of the specified interval. The uniform distribution is denoted by symbol $U[-,-]$ in Table 1 . The fundamental cost of operating QC is set as $c=100 \$ / \mathrm{hr}$. The desired berthing position of vessel $i(i \in \mathcal{V})$ is determined by generating a random integer distributed uniformly $U\left[0, L-l_{i}\right]$. The expected arrival time $t_{i}^{a}$ of vessel $i(i \in \mathcal{V})$ is assumed to follow a uniform distribution $U\left[0, T-\bar{t}_{i}\right]$, and its estimated departure time is set to $t_{i}^{d}=t_{i}^{a}+\bar{t}_{i}$, where $\bar{t}_{i}$ is the estimated midpoint value between $t_{i}^{\min }$ and $t_{i}^{\max }$, given by $\bar{t}_{i}=$ 
$\left\lceil\frac{t_{i}^{\min }+t_{i}^{\max }}{2}\right\rceil$, here $t_{i}^{\min }=\left\lceil\frac{w_{i}}{\left(N_{i}^{\max }\right)^{\widetilde{\alpha}}}\right\rceil$ and $t_{i}^{\max }=\left\lceil\frac{\left(1+\widehat{\alpha}\left(L-l_{i}\right)\right) w_{i}}{\left(N_{i}^{\min }\right)^{\tilde{\alpha}}}\right\rceil$. The energy consumption of QCs is set to $\mu=149.7 \mathrm{KWh} / \mathrm{hr}$ (Chang et al., 2010), and the carbon emission factor of a QC is set to $\alpha=$ $1.0935 \mathrm{~kg} / \mathrm{KWh}$ (Geerlings and Duin, 2011). Additionally, the interference exponent of QCs is set to $\tilde{\alpha}=0.9$, and the position deviation factor of vessels is set to $\hat{\alpha}=0.01$, which are both the same as those of the set in Meisel and Bierwirth (2009). The carbon emission is divided into $N$ segments ( $N$ is a random number not larger than 5), and the carbon taxation rate on each segment follows a uniform distribution $U[0.1,0.3 \times N]$ (unit: $\$ / \mathrm{kg}$ ). The dividing points $\Gamma_{S}$ are set to $\Gamma_{S}=50000 \times s$ $(s=1, \ldots, N-1), \Gamma_{N}$ is set to an extremely large number.

$<$ Table 1 is inserted here $>$

\subsection{Performance analysis}

We firstly linearize the piecewise objective function $C^{C T}(z)$ in Eq. (4) to obtain an integer linear programming model which is equivalent to Model 1, termed as Model 2 here. Therefore, we can solve the lexicographic optimization problems in Model 2 by CPLEX. Then, we evaluate the performance of our proposed solution from the two aspects stated above.

\subsubsection{Linearization of Model 1}

Let $y_{s}$ be binary variable for each segment $s(s=1, \ldots, N)$, and define $\bar{z}_{S}$ as a variable to represent the amount of carbon emission in segment $s$. Then, define $\mathbf{y}:=\left(y_{s}, s=1, \ldots, N\right)$ and $\overline{\mathbf{z}}:=$ $\left(\bar{z}_{s}, s=1, \ldots, N\right)$, and define the following carbon emission tax function:

$$
\mathscr{C} / \sigma^{T}(\mathbf{y}, \overline{\mathbf{z}}):=\sum_{s=1}^{N}\left(b_{s} y_{s}+\bar{z}_{s} r_{s}\right)
$$

Let $\mathbf{v}:=(\mathbf{x}, \mathbf{y}, \overline{\mathbf{z}})$ and define $\tilde{f}_{2}(\mathbf{v}):=C^{F C}(\mathbf{x})+\tilde{C}^{C T}(\mathbf{y}, \overline{\mathbf{z}})$. Then, we present Model 2 as below:

[Model 2]

$$
\min _{\mathbf{v}, z} f(\mathbf{v}, z)=\left(f_{1}(\mathbf{x}), \%_{2}^{\prime}(\mathbf{v})\right)
$$

s.t. (7)-(23), and

$$
\begin{gathered}
\sum_{s=1}^{N} \bar{z}_{s}=z \\
\sum_{s=1}^{N} y_{s}=1 \\
\Gamma_{s-1} y_{s} \leq \bar{z}_{s} \leq \Gamma_{s} y_{s} \quad \forall s \in\{1, \ldots, N\} \\
y_{s} \in\{0,1\} \quad \forall s \in\{1, \ldots, N\} \\
\bar{z}_{s} \geq 0 \quad \forall s \in\{1, \ldots, N\}
\end{gathered}
$$


where constraints (51)-(55) model the piecewise linear cost function.

Then, Model 1 is equivalently transformed into Model 2 and the lexicographic optimization problems in Model 2 can be solved by CPLEX.

\subsubsection{Performance Comparison between Heuristic ${ }^{\bar{F}^{1}}$ and CPLEX}

In order to compare Heuristic $\bar{F}^{1}$ with CPLEX on the computational performance, we generate four sets of test instances containing 10, 20,30, and 40 vessels and each set contains ten instances. All vessels in each instance are composed of $60 \%$ feeder vessels, $30 \%$ medium-sized vessels, and $10 \%$ jumbo vessels. The number of iteration Num in Heuristic ${ }^{\bar{F}^{1}}$ is set to 200. Here, we just implement Heuristic $^{\bar{F}^{1}}$ and CPLEX on each instance to obtain the nondominated point $F^{1}$ on the initial rectangle $\mathcal{R}:=\mathcal{R}((0,+\infty),(+\infty, 0))$, i.e. use them to solve the initial lexicographic optimization problem $F^{1}:=\operatorname{lexmin}\left\{F_{1}, F_{2}: F \in \mathcal{R}\right\}$. The computational performance of each instance solved by Heuristic $\bar{F}^{1}$ and CPLEX are shown in Table 2.

$<$ Table 2 is inserted here $>$

We firstly compare the computational performance between CPLEX and our heuristic. It can be seen from Table 2 that CPLEX is quite cumbersome to solve these instances, it delivers optimal solutions within a computational time of 10 hours only for 16 small-size instances in set 1 and set 2 . For all of the medium-size and large-size instances in set 3 and set 4, no optimal solutions can be found within 10 hours. When the instance size increases, the performance of CPLEX goes poorer. Conversely, the proposed solution algorithm is much more efficient than CPLEX. All instances in the four sets can be solved within a computational time of 10 minutes. Note that a number of lexicographic optimization models have to be solved in order to obtain the Pareto frontier of our proposed problem, hence, CPLEX is totally unacceptable for practical applications.

As for the quality of solutions obtained by CPLEX and Heuristic $\bar{F}^{1}$, we can see that the solutions to the 16 instances of set 1 and set 2 delivered by CPLEX are better than that of Heuristic $\bar{F}^{1}$, but the average relative gap between them are quite small. In set 1 , all instances can be solved by CPLEX within the computational time limit of 10 hours, and there are a total of five instances (\#1,\#2,\#4,\#6,\#10) that can be solved to the same optimality by both CPLEX and Heuristic $\bar{F}^{1}$. The average relative optimality gap of the first objective and the second objective between CPLEX and Heuristic $\bar{F}^{1}$ is 0.02 and 0.01 , respectively. In set 2, only two instances (\#7, \#9) can be solved to the same optimality by both CPLEX and Heuristic $\bar{F}^{1}$, and there are four instances 
$(\# 2, \# 5, \# 6, \# 8)$ that cannot be solved to optimality by CPLEX within computation time limit. The average relative optimality gap of the first objective and the second objective to six instances (\#1,\#3,\#4,\#7,\#9,\#10) between CPLEX and Heuristic ${ }^{\bar{F}^{1}}$ slightly increases to 0.05 and 0.02 , respectively. Therefore, the quality of solutions obtained by Heuristic $\bar{F}^{1}$ is acceptable in practice. Hence, Heuristic $\bar{F}^{1}$ is an effective and efficient solution algorithm to solve our proposed problem.

\subsubsection{Comparison of initial priority rule based on FCFS and operational productivity}

The initial priority rule proposed in Section 5.2.1 is based on the operational productivity required by vessels that can reflect vessel preferences for their preferred berth-QC plans. Now we can explore the role of the initial priority rule based on operational productivity in Heuristic $\bar{F}^{1}$, and compare it with the initial FCFS rule. Here, we implement the FCFS rule to get the initial priorities of vessels in Heuristic $\bar{F}^{1}$. The numerical results of the initial lexicographic optimization problem $F^{1}:=$ $\operatorname{lexmin}\left\{F_{1}, F_{2}: F \in \mathcal{R}\right\}$ solved by using the initial FCFS priority rule in Heuristic $\bar{F}^{1}$ are shown in Table 3.

$<$ Table 3 is inserted here $>$

In Table $3, \tilde{F}_{1}^{10}$ and $\widetilde{F}_{2}^{10}$ are initial solutions and $\widetilde{F}_{1}^{1 b}$ and $\tilde{F}_{2}^{1 b}$ are the best solutions to $F_{1}$ and $F_{2}$ found by the initial FCFS priority rule, respectively. $F_{1}^{10}$ and $F_{2}^{10}$ are initial solutions and $F_{1}^{1 b}$ and $F_{2}^{1 b}$ are the best solutions to $F_{1}$ and $F_{2}$ found by the initial operational productivity priority rule, respectively. Additionally, $G_{1}^{10}$ and $G_{2}^{10}$ are the relative differences between initial solutions $\tilde{F}_{1}^{10}$ and $F_{1}^{10}$, and $\tilde{F}_{2}^{10}$ and $F_{2}^{10}$, respectively, i.e. $G_{1}^{10}=\left(\tilde{F}_{1}^{10}-F_{1}^{10}\right) / F_{1}^{10}, G_{2}^{10}=$ $\left(\tilde{F}_{2}^{10}-F_{2}^{10}\right) / F_{2}^{10} . G_{1}^{1 b}$ and $G_{2}^{1 b}$ are the relative differences between best solutions $\tilde{F}_{1}^{1 b}$ and $F_{1}^{1 b}$, and $\tilde{F}_{2}^{1 b}$ and $F_{2}^{1 b}$, respectively, i.e. $G_{1}^{1 b}=\left(\tilde{F}_{1}^{1 b}-F_{1}^{1 b}\right) / F_{1}^{1 b}, G_{2}^{1 b}=\left(\tilde{F}_{2}^{1 b}-F_{2}^{1 b}\right) / F_{2}^{1 b}$.

The numerical results in Table 3 show that the initial solutions based on our proposed priority rule with operational productivities preferred by vessels are much better than those using the FCFS rule as the initial priority. It can be seen that in set 1 , the initial values to instance \#2 obtained by FCFS rule are 63 and 136.96, respectively, and they decrease to 36 and 81.52 by using the productivity rule with a decreasing rate of $77 \%$ and $68 \%$, respectively. It also can be seen that in set 1 , the productivity rule can improve the initial solutions at an average rate of $41 \%$ and $39 \%$, respectively. Similar results can be found with other instances as well. Therefore, the proposed rule to get the priority of vessels based on their preferred operational productivity is effective.

Table 4 also presents the computational time and the number of iterations required for the best 
solutions in Heuristic $\bar{F}^{1}$ under two initial priority rules. It can be seen that the initial priority rule under operational productivity not only takes less computational time compared with the initial priority under FCFS rule, but also requires less iterations. For instance, for Set 1, roughly an average $\mathrm{CPU}$ time of $6.08 \mathrm{~s}$ is required to get the best solution under the initial FCFS rule, and it requires about $4.09 \mathrm{~s}$ with the initial operational productivity rule. Now we focus on the influence of the different initial priority rules on the iteration process. The 10 samples in Set 1 run an average of about 4.3 iterations when the initial FCFS priority is applied. However, it is about 2.5 when the initial operational productivity rule is adopted. Obviously, a good initial solution effectively reduces the iteration number and the search performance of the algorithm can thereby be largely improved.

$<$ Table 4 is inserted here $>$

\subsection{Model analysis}

We analyze the model from the three aspects: the first is the economic analysis on operating costs, which focuses on the trade-off analysis between service efficiency and operating costs; the second is the environmental analysis on carbon emission; the third is the effect of varying tax rates and vessel estimated departure time on the trade-off.

\subsubsection{Economic analysis on operating costs}

In this subsection, the balanced box method is employed to obtain the Pareto frontier for each instance, and the trade-off between the service efficiency and operating costs of ports is investigated. Especially, the economic analysis on operating costs will be elaborated on.

The Pareto frontier for instance \#7 in set 1 is shown in Figure 4. It can be seen from Figure 4 that the Pareto frontier consists of seven nondominated points. The upper left point $(10,58.9)$ and the lower right point $(33,51.42)$ in the Pareto frontier corresponds to the preference of service efficiency and cost-saving, respectively. If the terminal planner adopts the berth plan indicated by the lower right point $(33,51.42)$, the port operator can reduce the operating costs from $58.9 \mathrm{k} \$$ to 51.42 $\mathrm{k} \$$, that is, $6.48 \mathrm{k} \$$ is saved, but at the expense of the increase of berthing waiting and departure delay from $10 \mathrm{~h}$ to $33 \mathrm{~h}$.

$<$ Figure 4 is inserted here $>$

The upper left point $(10,58.9)$ can be explained that the minimal operating costs involving fundamental cost and carbon emission taxation would be $58.9 \mathrm{k} \$$ subject to the constraint that the average berth waiting and departure delay for each vessel is not more than $1 \mathrm{~h}$ (one-hour berth waiting 
and departure delay limit). If the limit is relaxed to $1.5 \mathrm{~h}$, then the operating costs would decrease to $55.43 \mathrm{k} \$$, saving a cost of $2.47 \mathrm{k} \$$. Figure 4 clearly shows the decreased trend of operating costs with the relaxation of the service efficiency limit, which indicates the trade-off between the service efficiency and operating costs. Moreover, the points constituted in the Pareto frontier correspond to the berth-QC plans under different berthing wait and departure delay limits. If the port operator restricts the service efficiency of berthing wait and departure delay limit within one-hour, the berth-QC plan suggested by the point $(10,58.9)$ can be referred to.

Now we let $(10,58.9)$ be the benchmark solution, and then, we can get the differences of other solutions with the benchmark, which are listed in Table 5. As can be seen from Table 5 that the ratios of $\Delta F_{1} / F_{1}^{\text {ideal }}$ and $\Delta F_{2} / F_{2}^{\text {nadir }}$ both show an increasing trend but the former has a larger increasing rate than the latter, while $\Delta F_{2} / \Delta F_{1}$ shows a decreasing trend, which indicates that the benefit of saving operating cost for port operator is becoming marginal but at more expense of harm on the service efficiency of the terminal. Therefore, cost-saving is not always the preference for the port operator because the service efficiency may be harmed more. Another useful observation for the port operator is the average value of $\Delta F_{2} / \Delta F_{1}$, given as $0.62 \mathrm{k} \$$ in Table 5, which means that if the port operator aims to improve service efficiency by one hour, it has to incur a cost of $0.62 \mathrm{k} \$$ additionally. In addition, Table 5 informs the port operator of the best and worst service efficiencies it can provide for vessels, and corresponding operating costs it takes.

\section{$<$ Table 5 is inserted here $>$}

\subsubsection{Environmental analysis on carbon emission}

Now we investigate the environmental analysis of carbon emission in this section. The carbon emission of each point constituted in Pareto frontier for instance \#7 in set 1 is shown in Figure 5. As can be seen that the carbon emission in the upper left point $(10,58.9)$ is the most as $52383.02 \mathrm{~kg}$, and the carbon emission in the lower right point $(33,51.42)$ is the least as $51400.84 \mathrm{~kg}$, which reduces a total of $982.18 \mathrm{~kg}$ of carbon emission. But the level of service efficiency decreases as the berth waiting and departure delay increases from $10 \mathrm{~h}$ to $33 \mathrm{~h}$.

$<$ Figure 5 is inserted here $>$

Like the trade-off between service efficiency and operating costs shown by Figure 4 in section 6.2.1, Figure 5 also shows the trade-off between the service efficiency and carbon emission. It indicates that the port operator can improve the port service efficiency, but at the expense of carbon 
emission.

\subsubsection{Effects analysis of varying vessel estimated departure time and tax rates}

This section focuses on the analysis of effects of varying vessel estimated departure time and carbon emission tax rates on the trade-off between service efficiency and operating costs. Here we set three different levels for the estimated turnaround time: tight, average and loose. The estimated departure time for vessels corresponding to the three levels of turnaround time are set as $t_{i}^{\text {tight }}=$ $t_{i}^{\min }, t_{i}^{\text {ave }}=\bar{t}_{i}$ and $t_{i}^{\text {loose }}=t_{i}^{\max }$, respectively. Similarly, in order to investigate the effect of varying tax rates, we set three levels for the carbon taxation rate: low, medium and high. In the low level, the carbon emission taxation rate on each segment follows a uniform distribution $U[0,0.1 \times N]$; in the medium level, it follows a uniform distribution $U[0.1 \times N, 0.2 \times N]$; and in the high level, it follows a uniform distribution $U[0.2 \times N, 0.3 \times N]$. Four test instances containing 10, 20, 30 and 40 vessels are generated, and labeled with the number of \#41 \#44, respectively, in order to distinguish them with the former instances. Similar with Section 6.1.2, all vessels in each instance are composed of $60 \%$ feeder vessels, $30 \%$ medium-sized vessel, and $10 \%$ jumbo vessels. All other data for vessels, such as their length, workload, desired berthing position and expected arrival time are generated in the same way described before. The Pareto frontiers for the four test instances are depicted in Figure 6Figure 9, respectively.

\footnotetext{
$<$ Figure 6 is inserted here $>$

$<$ Figure 7 is inserted here $>$

$<$ Figure 8 is inserted here $>$

$<$ Figure 9 is inserted here $>$
}

As can be seen from Figure 6 - Figure 9, we can find that the Pareto frontiers corresponding to the three levels of varying estimated departure time has a rightward trend on the value of $F_{1}$, namely the service efficiency. The rationale behind is that the estimated turnaround time is becoming tighter and tighter, which has negative impact on the service efficiency. Also, we can find that the Pareto frontiers corresponding to the three levels of varying carbon emission taxation rates in each instance has an upward trend on the value of $F_{2}$, namely the total costs of fundamental cost and carbon taxes. Obviously, it is reasonable because the carbon emission taxation rates are becoming higher and higher. An interesting finding is that the Pareto frontiers for the three levels in each instance have the same 
number of nondominated points. For example, there are 6 nondominated points in the Pareto frontiers in instance \#41. It can also find that the number of nondominated points in the Pareto frontiers for different instances are different, and becomes more in instances with more vessels. The number of nondominated points increases from 6 to 14 in instance \#41 to instance \#44.

The analysis above focuses on the joint effects of varying vessel estimated departure time and carbon emission tax rates on the trade-off between service efficiency and operating costs. Now we analyze them separately. Firstly, we observe the effect of varying vessel estimated departure time on the trade-off between service efficiency and operating costs. We adopt the same methodology above to set three different levels for the estimated turnaround time, and generate four test instances containing 10,20, 30 and 40 vessels labeled with the number of \#45 \#48, respectively. The Pareto frontiers for these four test instances are depicted in Figure 10- Figure 13, respectively. They illustrate that with the shrink of the estimated turnaround time of vessels moored at port, the service efficiency of port deteriorates, but the operating costs are not influenced. It tells us that: 1) not only the plan of berth allocation and QC assignment affects the service efficiency of port, the estimated turnaround time of vessels moored at port affects it as well; 2) the service efficiency of port may be different even if the port operator takes the same operating costs. Another valuable finding is that the estimated turnaround time of vessels moored at port also influences the number of nondominated points in the Pareto frontiers. For example, 7 points are obtained for loose level, but there are both 8 points for medium and tight levels. It is reasonable and we can explain it because the upper left points for loose and medium level have the same service efficiency but with different operating costs. We can see that the service efficiency are both 0 , but the operating costs in medium level are higher than that in loose level.

\section{$<$ Figure 10 is inserted here $>$ \\ $<$ Figure 11 is inserted here $>$ \\ $<$ Figure 12 is inserted here $>$ \\ $<$ Figure 13 is inserted here $>$}

We now explore the effect of carbon emission tax rates on the trade-off between service efficiency and operating costs. We still adopt the same methodology above to set three different levels for the rates, and generate another four instances labeled with the number of \#49 \#52, respectively. The Pareto frontiers for the four instances are depicted in Figure 14- Figure 17, respectively. And they 
illustrate that with the increase of the carbon emission tax rates, more operating costs are incurred to port operator, but service efficiency of port are not influenced. Similarly, it tells us that: 1) not only the plan of berth allocation and QC assignment affects the operating costs of port, the carbon emission tax rates affects it as well; 2) the service efficiency of port may be the same even if the port operator takes different operating costs. However, unlike the estimated turnaround time, the taxation rates cannot influence the number of nondominated points in the Pareto frontiers. We can see that in each figure, there are the same amount of nondominated points in the Pareto frontiers for different levels of taxation rates. It is reasonable as well and we can explain it because the lower right points in the Pareto frontiers for different levels of taxation rates never have operating costs of 0 but with different service efficiency.

\author{
$<$ Figure 14 is inserted here $>$ \\ $<$ Figure 15 is inserted here $>$ \\ $<$ Figure 16 is inserted here $>$ \\ $<$ Figure 17 is inserted here $>$
}

\title{
7. Conclusion
}

This paper studies the trade-off between the service efficiency and operating costs for the joint BAQCAP. The service efficiency is measured by the berth waiting and departure delay time of vessels, and the operating costs contain the fundamental cost and carbon emission taxation. A BOIP model is proposed for the problem, and the balanced box method developed by Boland et al. (2015) is employed here to cope with the biobjectives. In order to solve the lexicographic optimization models generated in the balanced box method, an efficient heuristic algorithm is designed based on the analysis and properties of the BIOP model. Then, numerical experiments are carried on to evaluate the applicability and effectiveness of the proposed models and solution algorithm.

Our future work will focus on the three aspects. First, it would be interesting to extend the current berth-QC plan to integrated terminal plan, because the current berth-QC plan only considers the operations between vessels and shore platform, but the operations between the shore platform and storage yard are out of consideration. Actually, container trucks (CTs) which deliver containers between shore platform and yard, and yard cranes (YCs) which operate the containers in yard, both contribute to carbon emission in the terminal as well. Therefore, an integrated terminal plan involving berth allocation, QC assignment, CT deployment and YC assignment needs to be studied. The second 
is to consider the variation of the taxation cost function studied in this paper. Here the taxation cost function is assumed to be piecewise linear. If it is not piecewise linear, we can approximate it by a piecewise linear function. The problem is that how many segments should be adopted or how many points should be chosen in order to keep the approximation error within an acceptable level, which is also another future research topic of interest to us. The third is to study the cooperation between vessels and port operators. Within the cooperation, vessels can change their scheduled arrival time and departure time, which may benefit port operators but cause negative consequence for vessels. How to design a win-win cooperation pattern for vessels and port operators is an interesting research topic.

\section{Acknowledgement}

The authors appreciate the associate editor and two anonymous reviewers for their suggestions and comments. This research is partially supported by the National Natural Science Foundation of China [No. 71771180, 71725007, 71525003,71890974/71890970] and Humanities and Social Science Foundation of Ministry of Education of China [No. 16YJC630112].

\section{References}

Agra, A., Oliveira, M., 2018. MIP approaches for the integrated berth allocation and quay crane assignment and scheduling problem. European Journal of Operations Research, 264(1), 138-148.

Aneja, Y.P., Nair, K.P.K., 1979. Bicriteria transportation problem. Management Science, 27, 73-78.

Bierwirth, C., Meisel, F., 2010. A survey of berth allocation and quay crane scheduling problems in container terminals. European Journal of Operational Research, 202 (3), 615-627.

Bierwirth, C., Meisel, F., 2015. A follow-up survey of berth allocation and quay crane scheduling problems in container terminals. European Journal of Operational Research, 244(202), 615-627.

Bowman, V.J., 1976. On the relationship of the Tchebycheff norm and the efficient frontier of multiple-criteria objectives. Thieriez, H., Zionts, S. eds. Multiple Criteria Decision Making, Springer Verlag, Berlin, 76-85.

Boland, N., Charkhgard, H., Savelsbergh, M., 2015. A criterion space search algorithm for biobjective integer programming: the balanced box method. INFORMS Journal on Computing, 27(4), 735-754.

Chalmet, L., Lemonidis, L., Elzinga, D., 1986. An algorithm for the bi-criterion integer programming problem. European Journal of Operational Research, 25(2), 292-300.

Chang, D., Jiang, Z., Yan, W., He, J., 2010. Integrating berth allocation and quay crane assignments. 
Transportation Research Part E, 46(6), 975-990.

Chankong, V., Haimes, Y.Y., 1983. Multiobjective Decision Making Theory and Methodology, Elsevier Science, New York.

Croxton, K.L., Gendron, B., Magnanti, T., 2003. A comparison of mixed-integer programming models for nonconvex piecewise linear cost minimization problems. Manage Science. 49 (9), 1268-1273.

Du, Y., Chen, Q., Quan, X., Long, L., Fung, R. Y. K., 2011. Berth allocation considering fuel consumption and vessel emissions. Transportation Research Part E, 47(6), 1021-1037.

Flues, F., Lutz, B.J., 2014. The effect of electricity taxation on the German manufacturing sector: a regression discontinuity approach. https://www/econstor.eu/bitstream/ 10419/107421/1/818759607.pdf. Accessed on Jan. 29, 2018.

Geerlings, H., Duin, R.V., 2011. A new method for assessing CO2-emissions from container terminals: a promising approach applied in Rotterdam. Journal of Cleaner Production, 19(6-7), 657-666.

Giallombardo, G., Moccia, L., Salani, M., Vacca, I., 2010. Modeling and solving the tactical berth allocation problem. Transportation Research Part B, 44, 232-245.

Guan, Y., Cheung, R.K., 2004. The berth allocation problem: models and solution methods. OR Spectrum, 26(1), 75-92.

He, J., 2016. Berth allocation and quay crane assignment in a container terminal for the trade-off between time-saving and energy-saving. Advanced Engineering Informatics, 30(3), 390-405.

Hu, Q. M., Hu, Z. H., Du, Y., 2014. Berth and quay-crane allocation problem considering fuel consumption and emissions from vessels. Computers \& Industrial Engineering, 70(4), 1-10.

Imai, A., Chen, H. C., Nishimura, E., Papadimitriou, S., 2008. The simultaneous berth and quay crane allocation problem. Transportation Research Part E, 44(5), 900-920.

IMO. Interim guidelines for voluntary ship CO2 emission indexing for use in trails, MEPC/Circ. 471, 29 July 2005.

Iris, C., Pacino, D., Ropke, S., Larsen, A., 2015. Integrated berth allocation and quay crane assignment problem: set portioning models and computational results, Transportation Research Part E, 81, 75-97.

Iris, C, Pacino, D., Ropke, S., 2017. Improved formulations and an adaptive large neighborhood search heuristic for the integrated berth allocation and quay crane assignment problem. 
Transportation Research Part E, 105, 123-147.

Jelenić, T.A., 2016. The green port policy: Why \& What, http://www.theicct.org/sites/default/files/Thomas\%20Jelenic_Greenport_En.pdf

Kim, K.H., Moon, K.C., 2003. Berth scheduling by simulated annealing. Transportation Research Part B, 37(6), 541-560.

Lee, D.H., Chen, J., Cao, J., 2010. The continuous Berth Allocation Problem: A Greedy Randomized Adaptive Search Solution, Transportation Research Part E, 46(6), 1017-1029.

Li, F., Sheu, J., Gao, Z., 2015. Solving the continuous berth allocation and specific quay crane assignment problems with quay crane coverage range. Transportation Science, 49(4), 968-989.

Lim, A., 1998. The berth planning problem. Operations Research Letters, 22 (2-3), 105-110.

Liu, M., Lee, C-Y., Zhang, Z., Chu, C., 2016. Bi-objective optimization for the container terminal integrated planning. Transportation Research Part B, 93, 720-749.

Mshe, M.P, 2012. Reducing air emissions in the port of Rotterdam, http://www.theicct.org/sites/default/files/Maurits\%20Prinssen.pdf

Meisel, F., Bierwirth, C., 2009. Heuristics for the integration of crane productivity in the berth allocation problem. Transportation Research Part E, 45(1), 196-209.

Park, Y. M., Kim, K. H., 2003. Container terminals and automated transport systems. Operations Research-Spectrum, 25(1), 1-23.

Port of NY and NJ, 2009. A clean air strategy for the port of New York and New Jersey, https://www.panynj.gov/about/pdf/CAS-FINAL.pdf

Schaefer, J. M., 1969. Clothing Exemptions and Sales Tax Regressivity. The American Economic Review. 59 (4), 596-599.

Schonfeld, P., Sharafeldien, O., 1985. Optimal berth and crane combinations in containerports. Journal of waterway, port, coastal and ocean engineering, 111(6), 1060-1072.

Shang, X., Cao, J., Ren, J., 2016. A robust optimization approach to the integrated berth allocation and quay crane assignment problem. Transportation Research Part E, 94, 44-65.

Steuer, R.E., Choo, E., 1983. An interactive weighted Tchebycheff procedure for multiple objective programming. Mathematical Programing, 26(3), 326-344.

Stidsen, T., Andersen, K.A., Dammann, B., 2014. A branch and bound algorithm for a class of biobjective mixed integer programs. Management Science, 60(4), 1009-1032. 
Vacca, I., Salani, M., Bierlaire, M., 2013. An exact algorithm for the integrated planning of berth allocation and quay crane assignment. Transportation Science, 47 (2), 148-161.

Wang, K., Zhen, L., Wang, S., Laporte, G., 2018a. Column generation for the integrated berth allocation, quay crane assignment, and yard assignment problem. Transportation Science, 52(4), 812-834.

Wang, T., Wang, X., Meng, Q., 2018b. Joint berth allocation and quay crane assignment under different carbon taxation policies. Transportation Research Part B, 117, 18-36.

$\mathrm{Xu}, \mathrm{Z}$., Lee, C-Y., 2018. New lower bound and exact method for the continuous berth allocation problem. Operations Research, 66(3), 778-798.

Zhen, L., Liang, Z., Dan, Z., Lee, L.H., Chew, E.P., 2017. Daily berth allocation in a tidal port with channel flow control. Transportation Research Part B, 106, 193-217. 


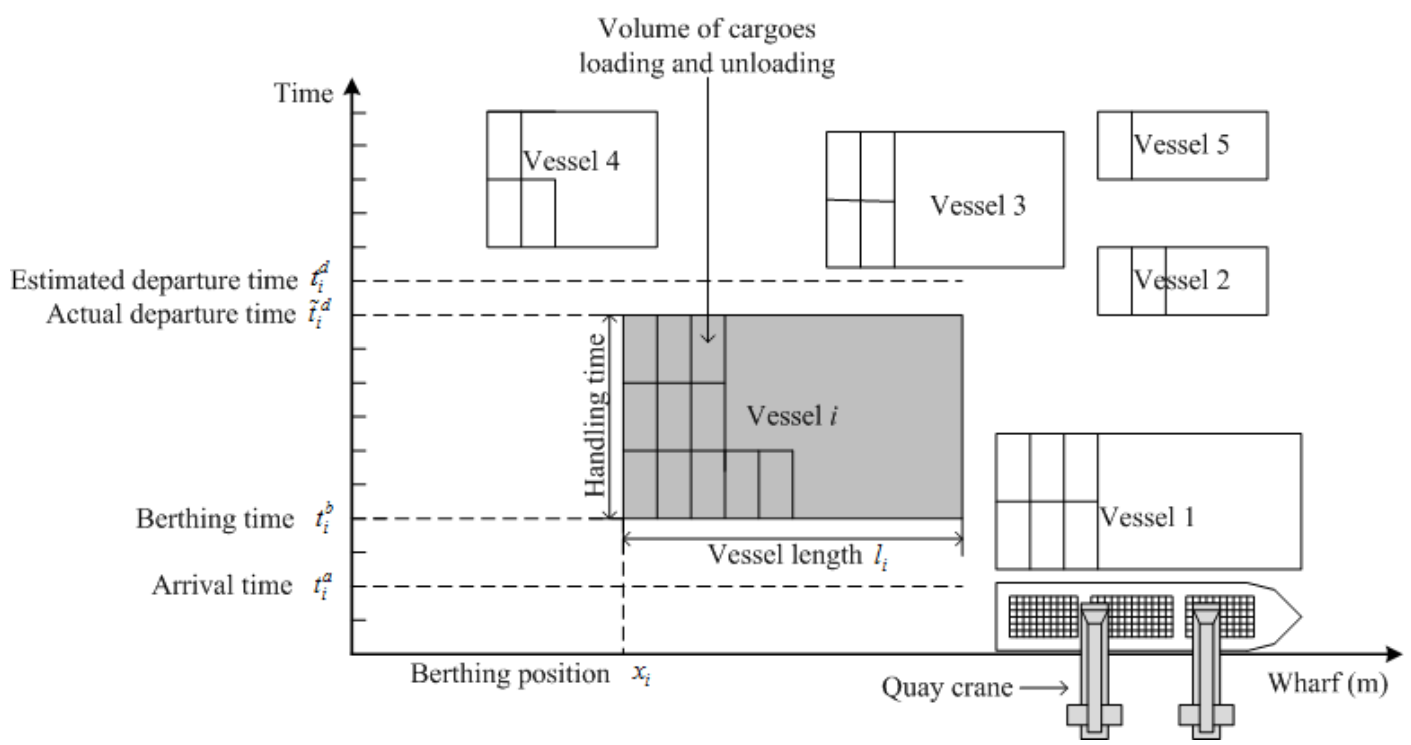

Figure 1. Illustration of BACAP

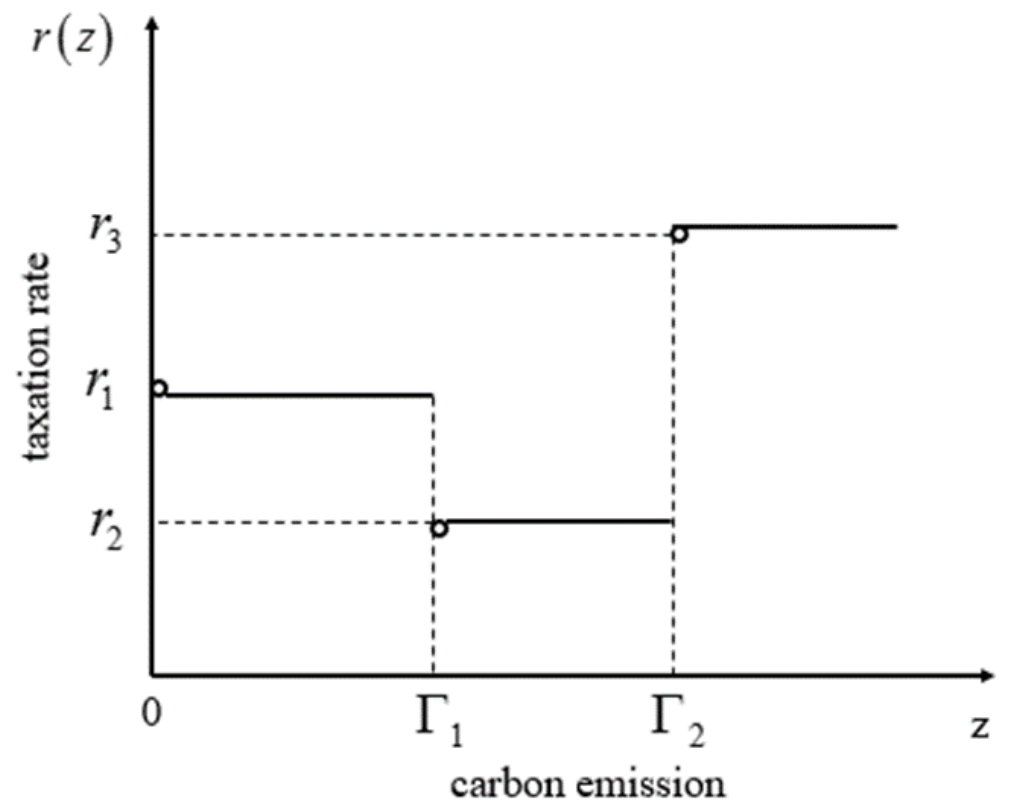

Figure 2. The general piecewise function of carbon emission taxation rate 


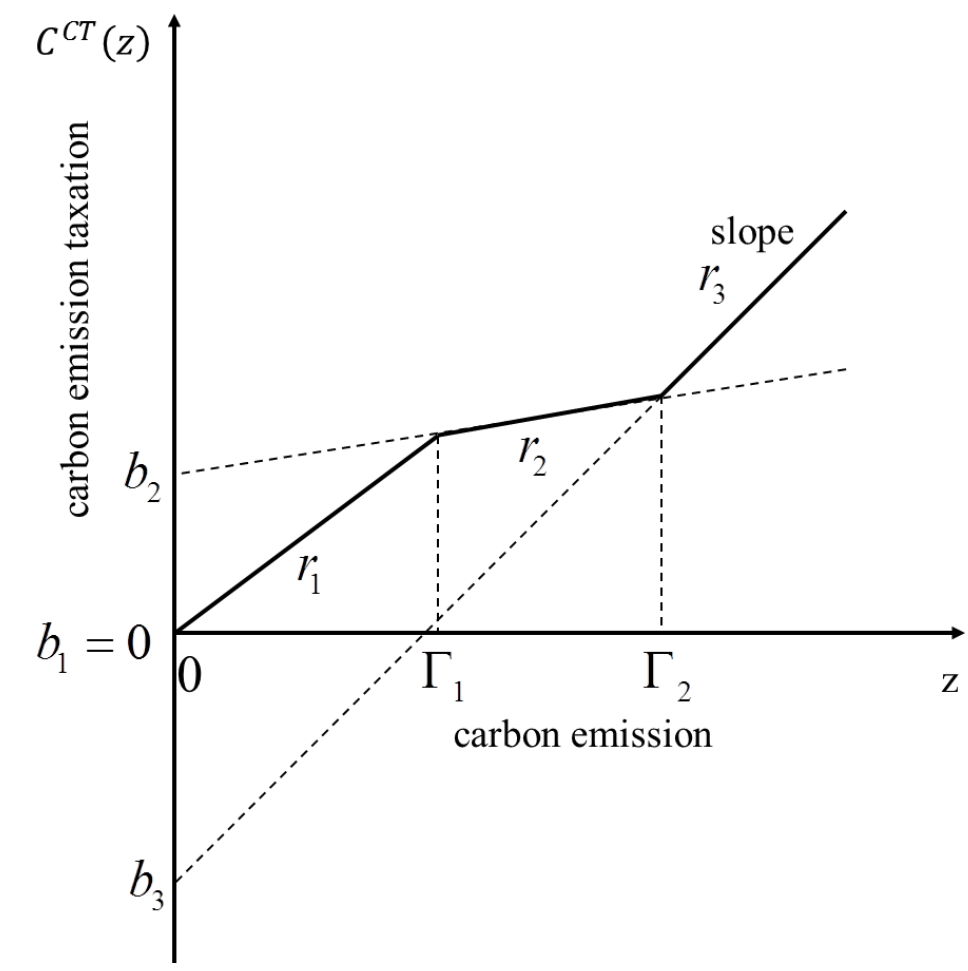

Figure 3. The general piecewise linear cost function of carbon emission taxation

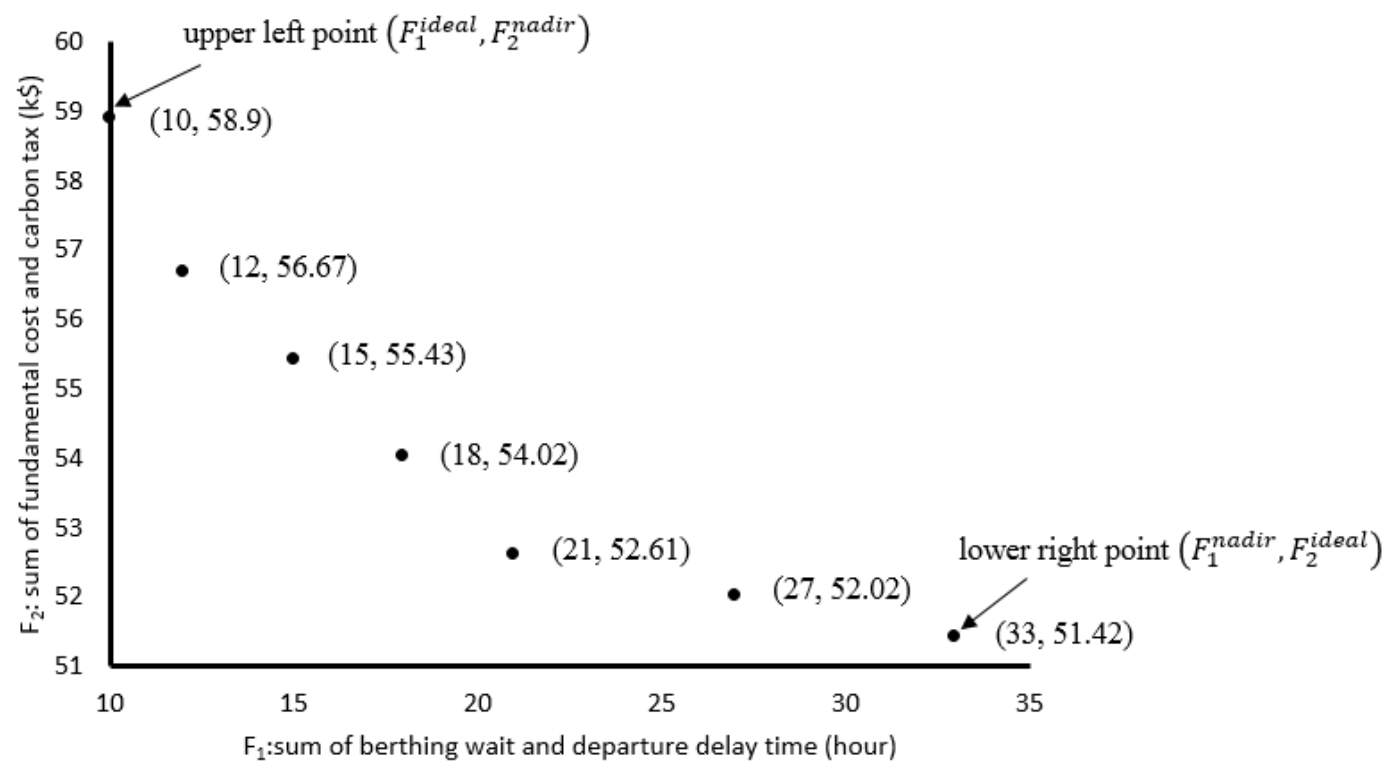

Figure 4. Pareto frontier for instance \#7 in set 1 (10 vessels/48 hours) 


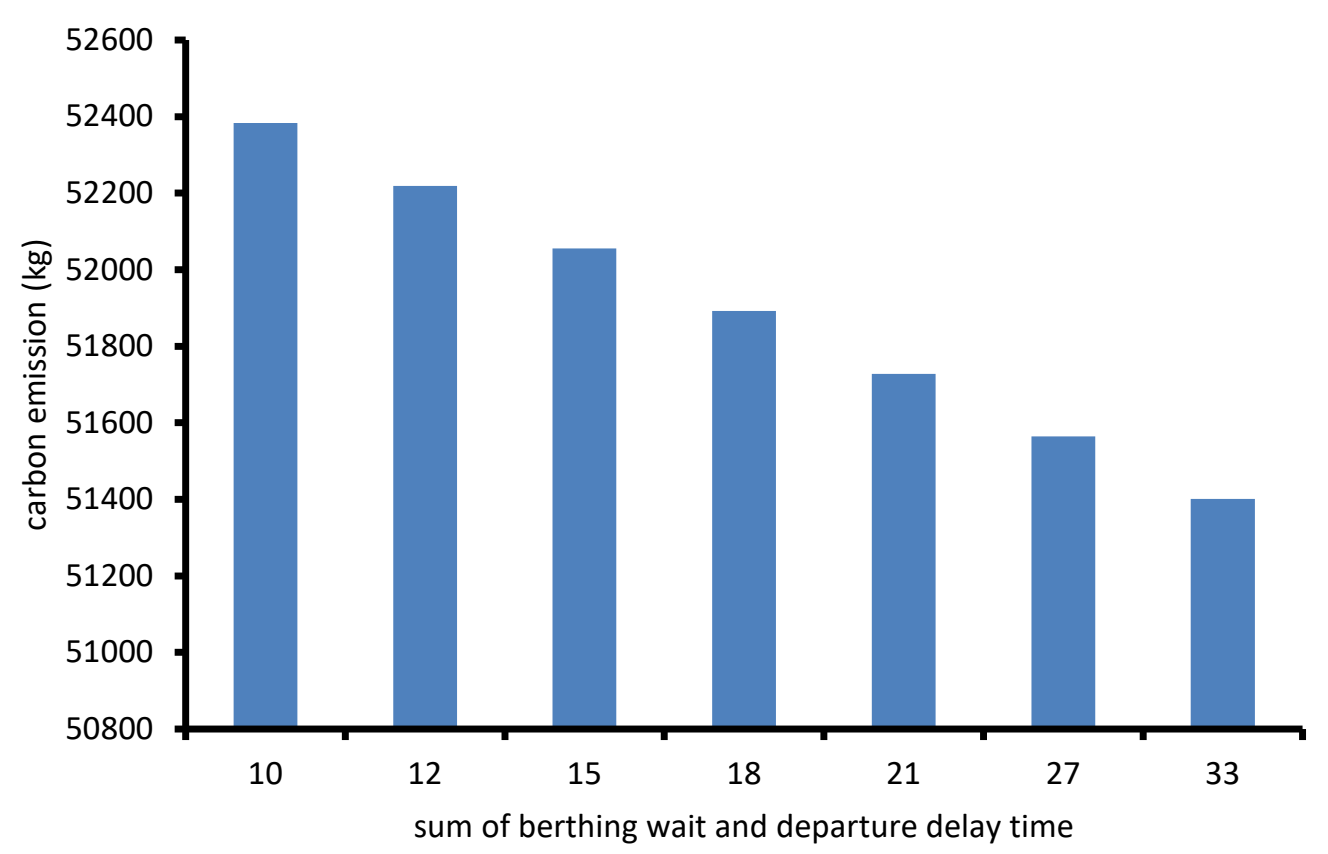

Figure 5. Carbon emission for instance \#7 in set 1 (10 vessels/48 hours)

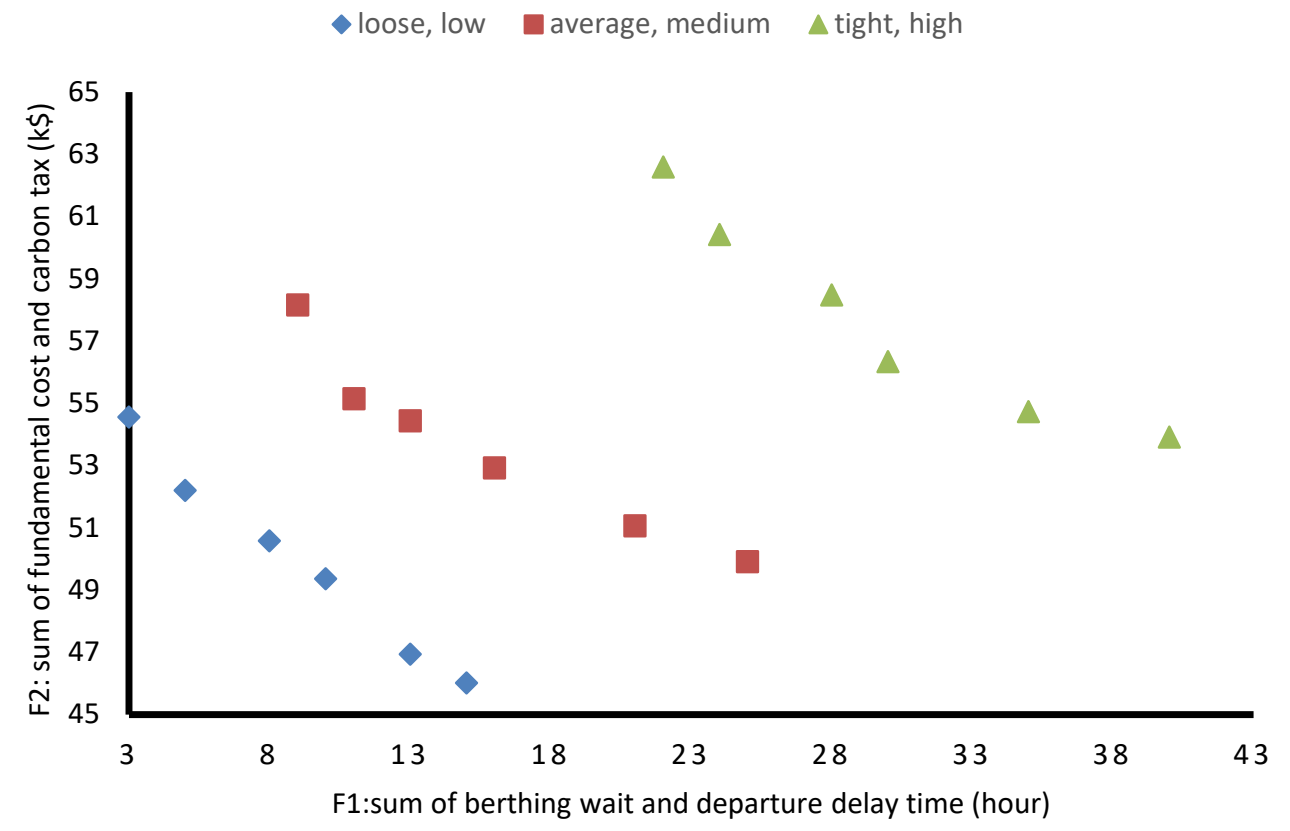

Figure 6. Pareto frontier for instance \#41 (10 vessels/48 hours) 


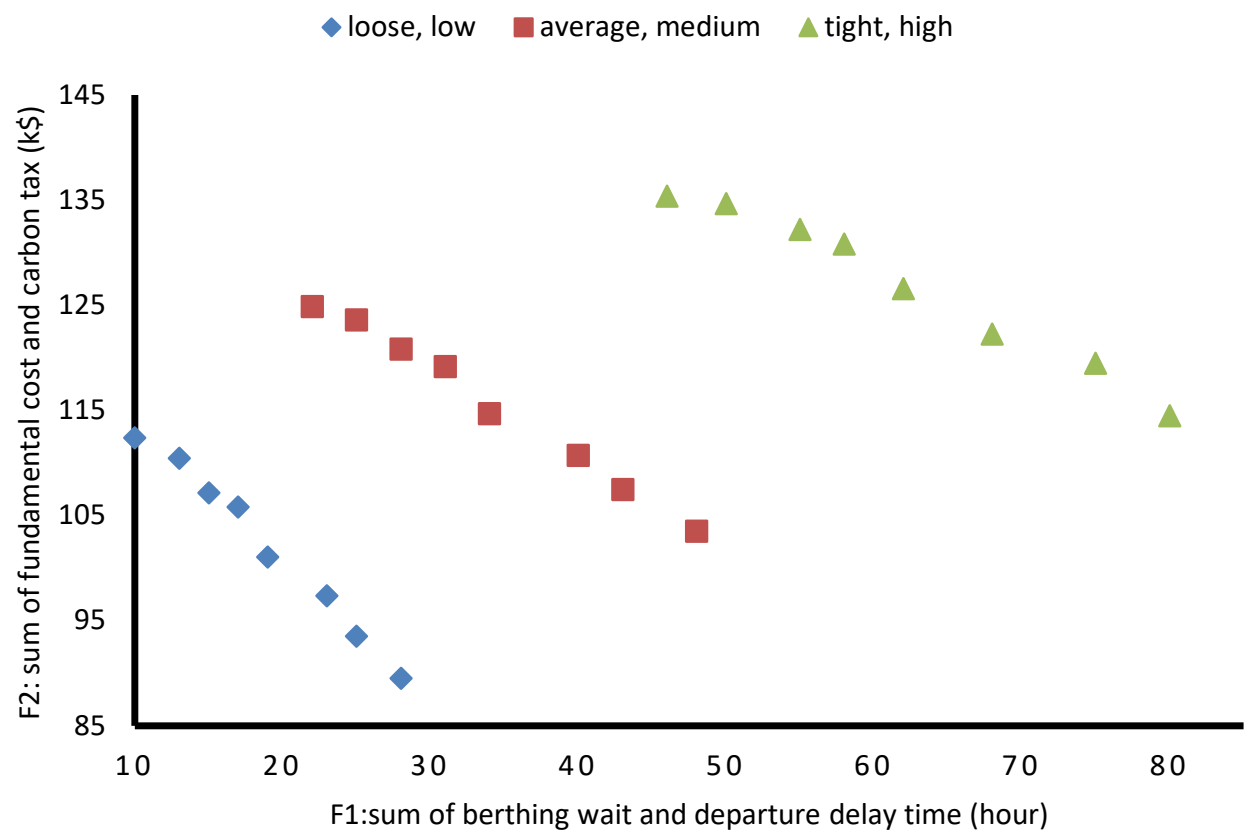

Figure 7. Pareto frontier for instance $\# 42$ (20 vessels/72 hours)

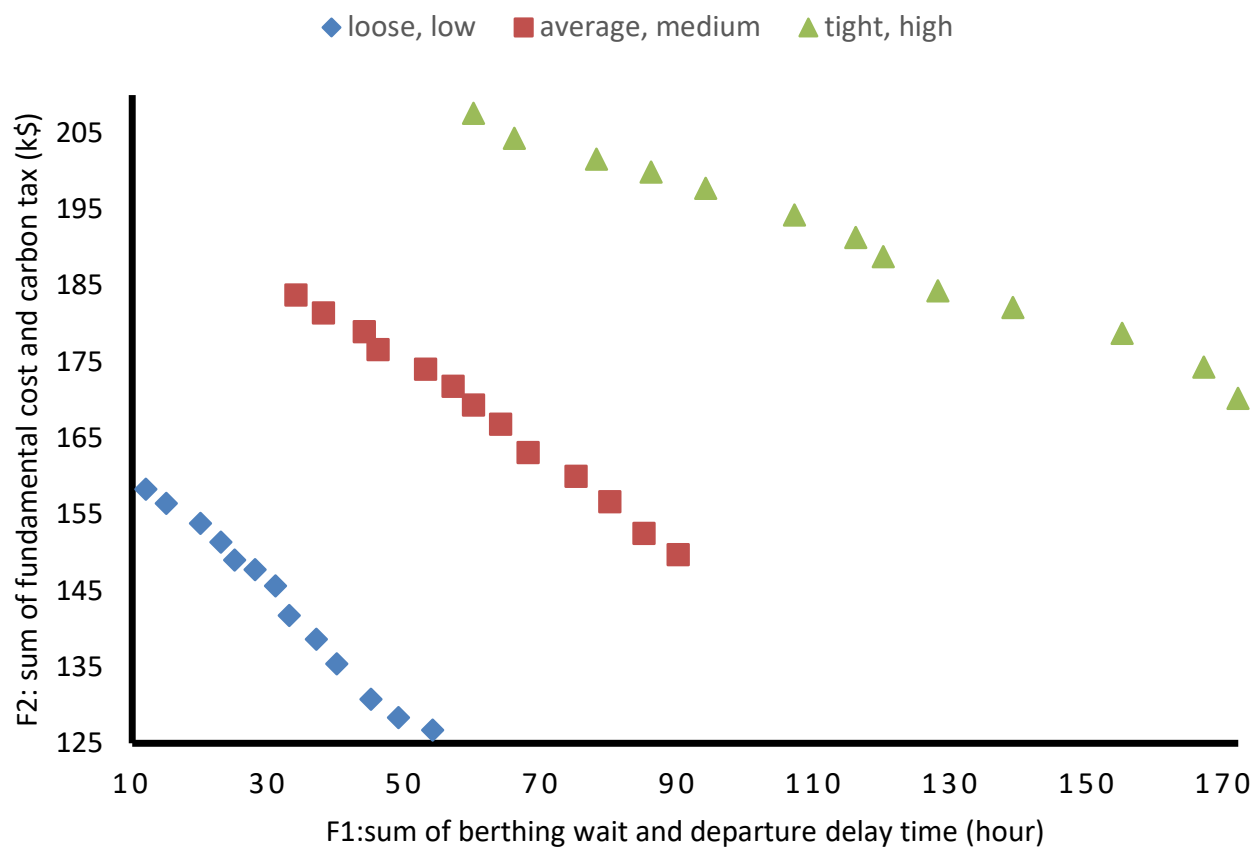

Figure 8. Pareto frontier for instance $\# 43$ (30 vessels/96 hours) 


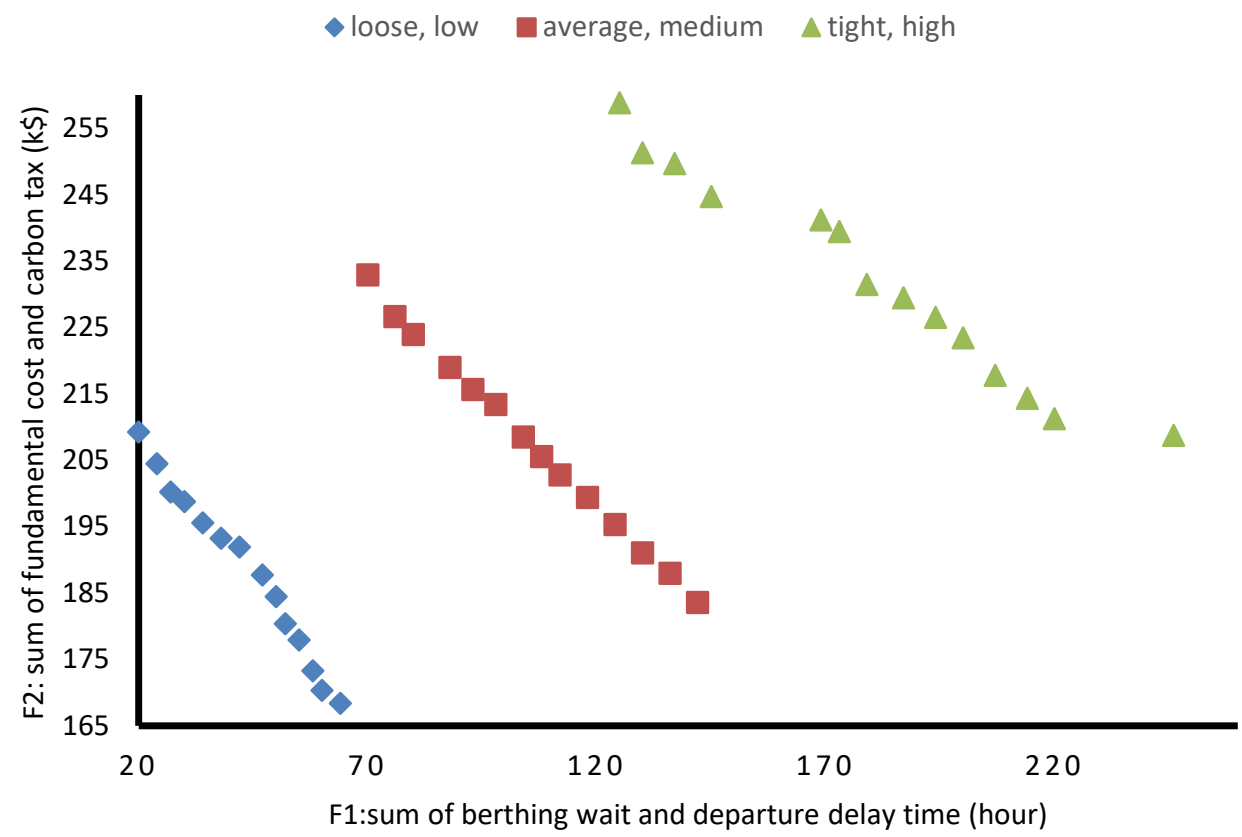

Figure 9. Pareto frontier for instance \#44 (40 vessels/168 hours)

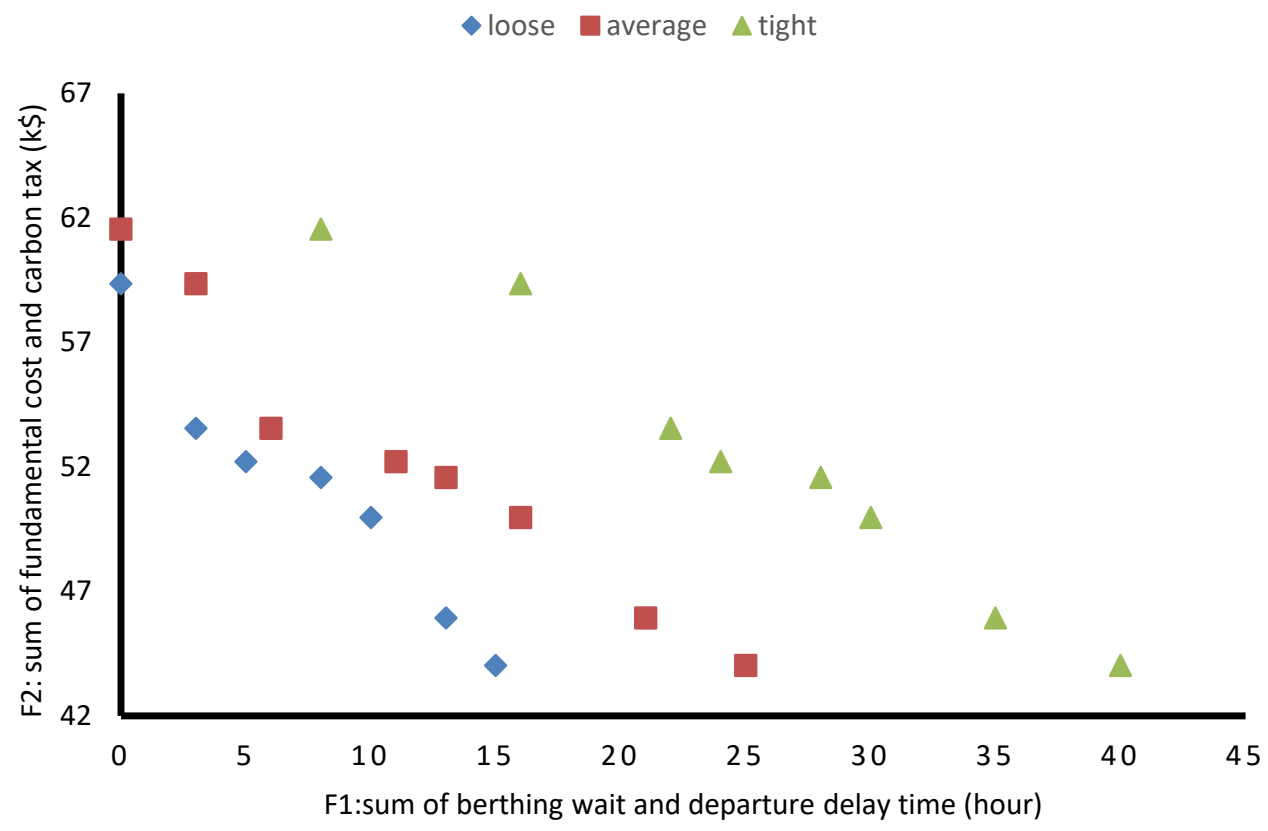

Figure 10. Pareto frontier for instance \#45 (10 vessels/48 hours) 


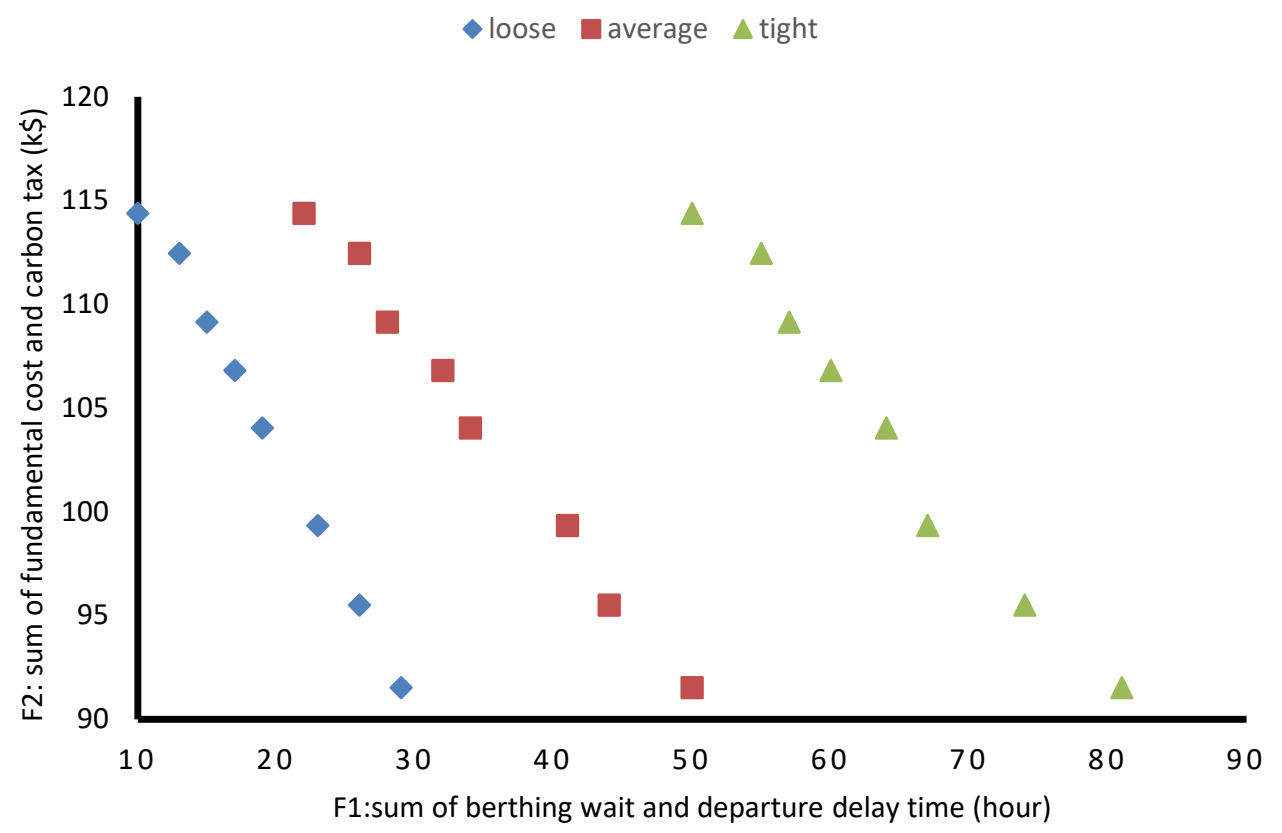

Figure 11. Pareto frontier for instance \#46 (20 vessels/72 hours)

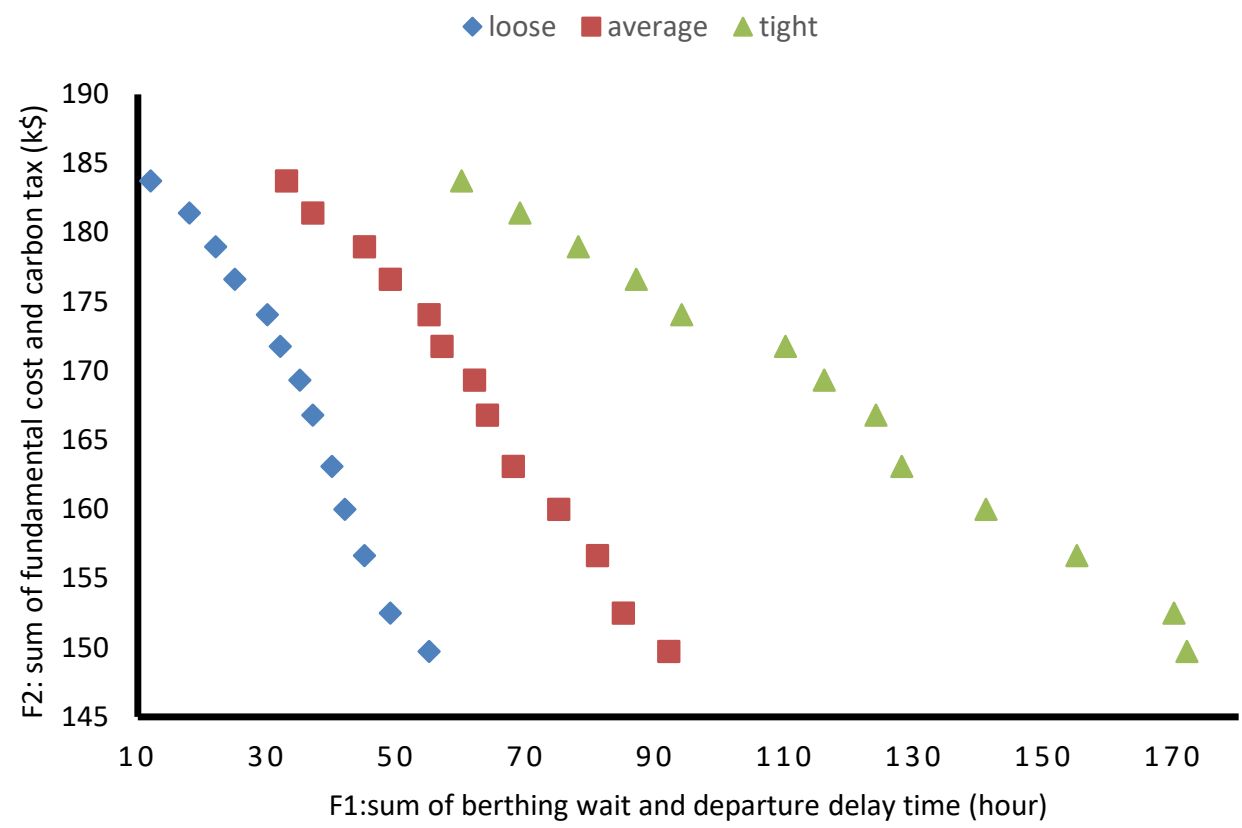

Figure 12. Pareto frontier for instance \#47 (30 vessels/96 hours) 


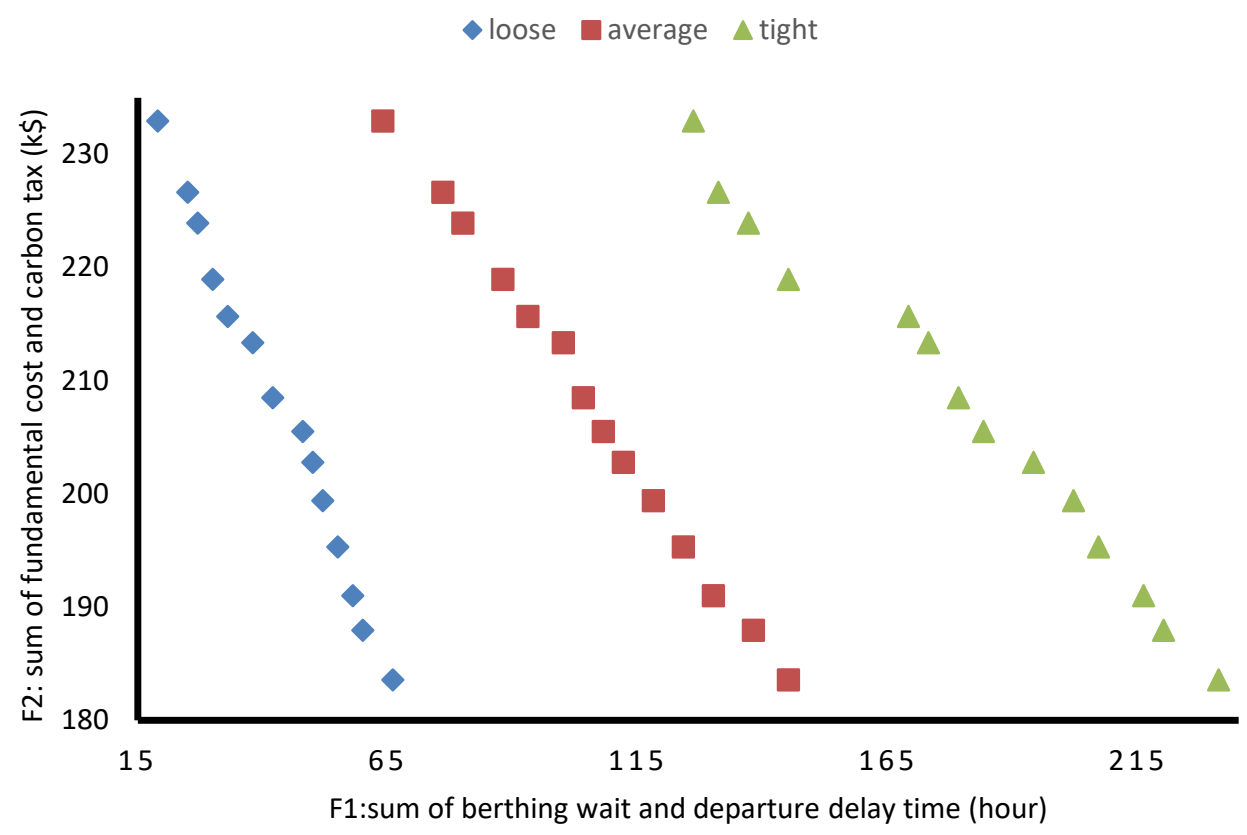

Figure 13. Pareto frontier for instance $\# 48$ (40 vessels/168 hours)

$\diamond$ low medium $\Delta$ high

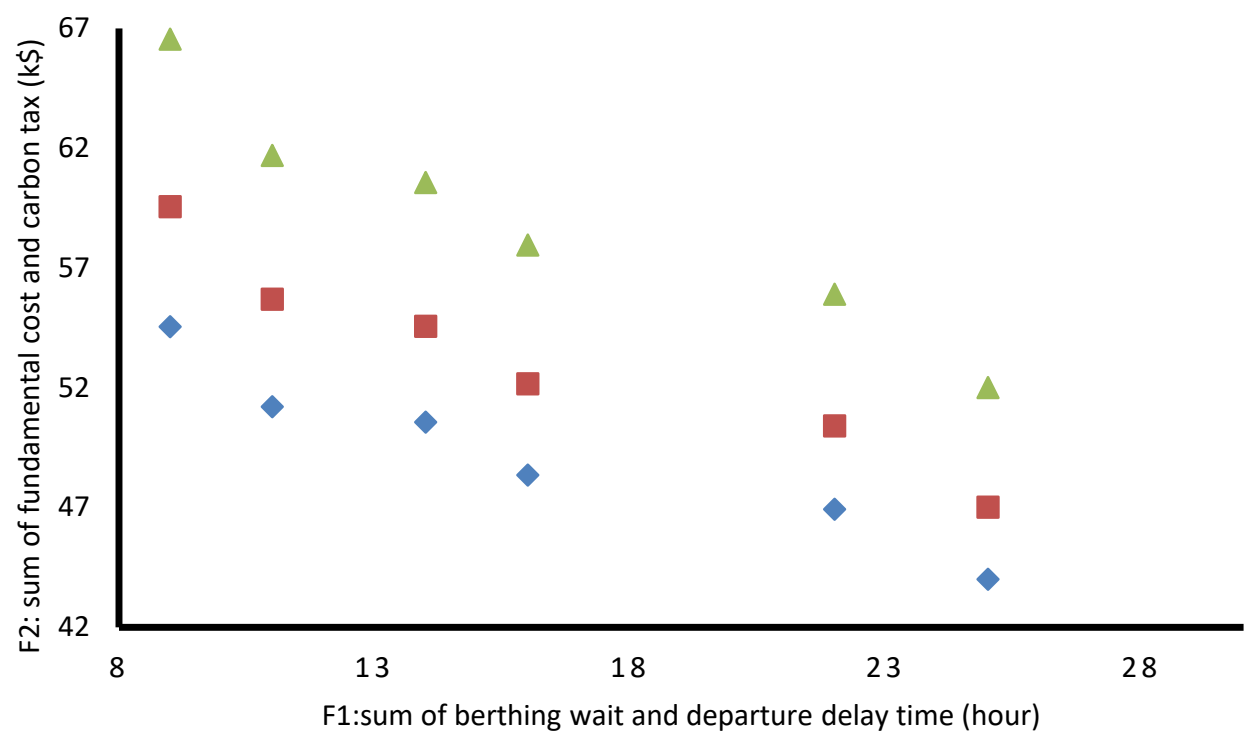

Figure 14. Pareto frontier for instance \#49 (10 vessels/48 hours) 


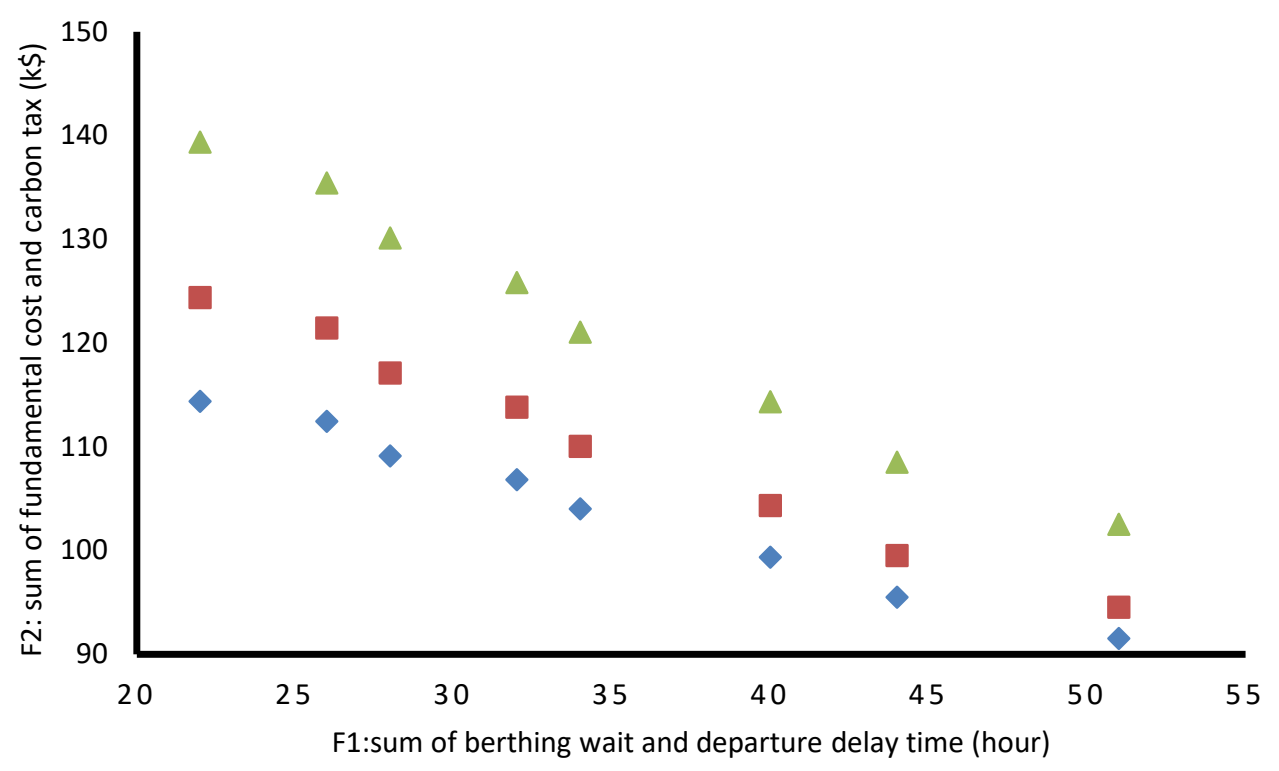

Figure 15. Pareto frontier for instance \#50 (20 vessels/ 72 hours)

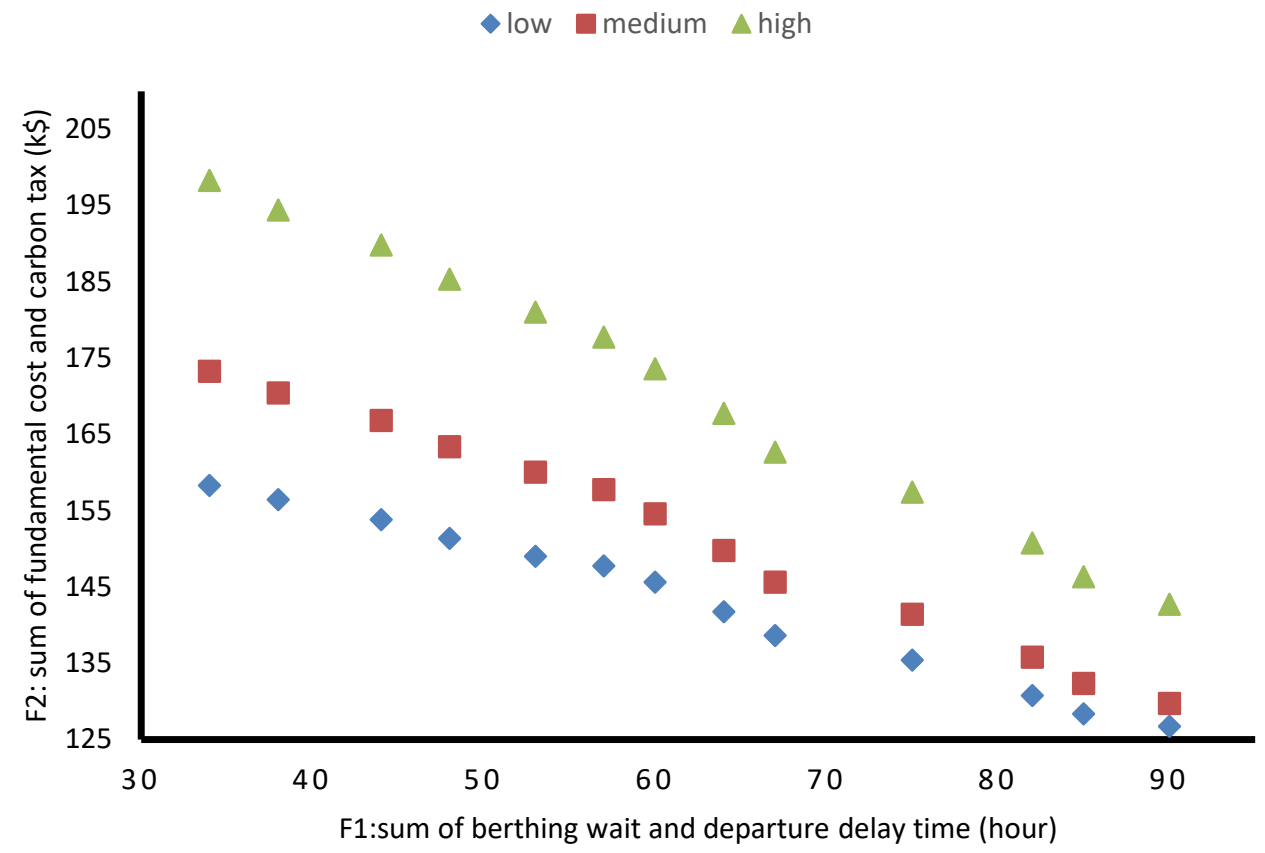

Figure 16. Pareto frontier for instance \#51 (30 vessels/96 hours) 
$\checkmark$ low amedium $\triangle$ high

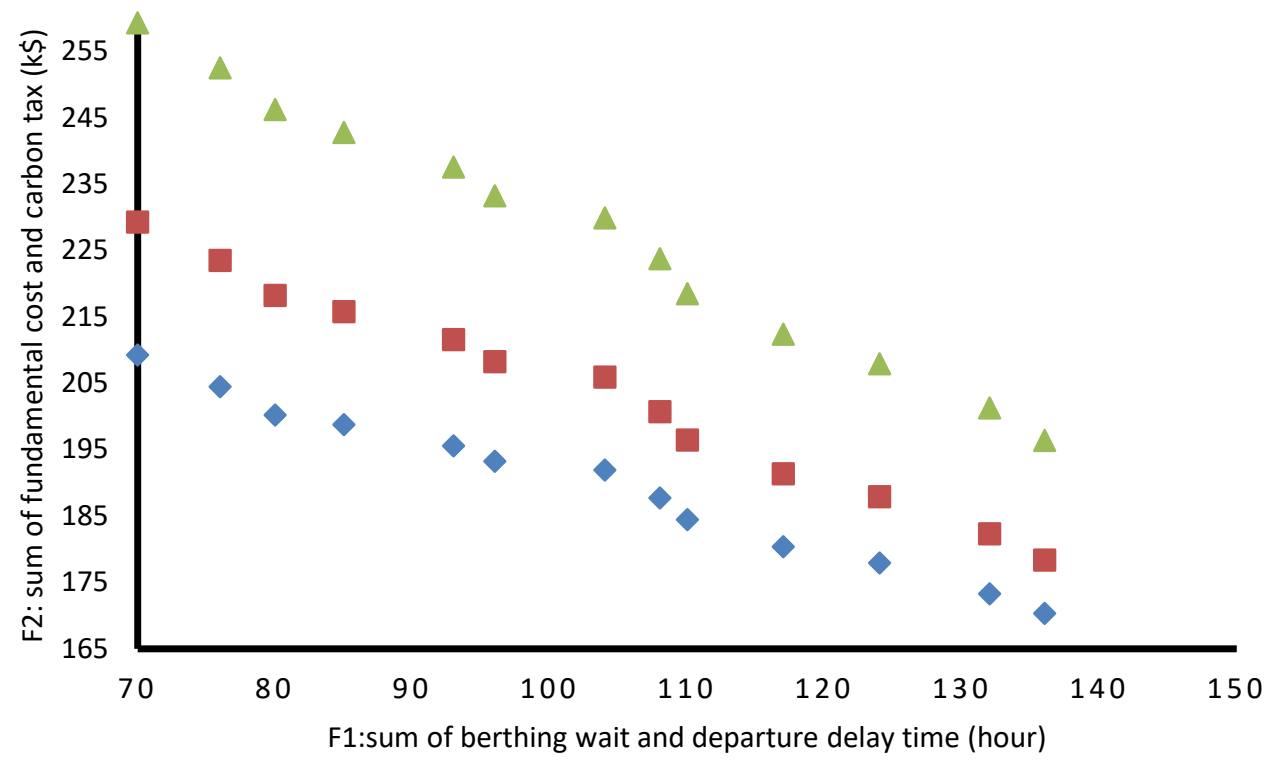

Figure 17. Pareto frontier for instance \#52 (40 vessels/168 hours) 
Table 1. Technical specifications for different vessel classes

\begin{tabular}{lllcc}
\hline Class & $l_{i}(\mathrm{~m})$ & $W_{i}(\mathrm{QC}-\mathrm{hr})$ & $N_{i}^{\min }$ & $N_{i}^{\max }$ \\
\hline Feeder & $\mathrm{U}[80,210]$ & $\mathrm{U}[5,15]$ & 1 & 2 \\
Medium & $\mathrm{U}[210,300]$ & $\mathrm{U}[15,30]$ & 2 & 4 \\
Jumbo & $\mathrm{U}[300,400]$ & $\mathrm{U}[30,40]$ & 4 & 6 \\
\hline
\end{tabular}

Data source: Meisel and Bierwirth (2009)

Table 2 Comparison between Heuristic $\bar{F}^{1}$ and CPLEX on initial lexmin $\left\{F_{1}, F_{2}: F \in \mathcal{R}\right\}$

\begin{tabular}{|c|c|c|c|c|c|c|c|c|c|c|c|c|c|c|c|c|}
\hline & \multicolumn{8}{|c|}{ Set 1: $V=10$ vessels, $T=48$ hours } & \multicolumn{8}{|c|}{ Set $2: V=20$ vessels, $T=72$ hours } \\
\hline & \multicolumn{4}{|c|}{ CPLEX (10 h) } & \multicolumn{4}{|c|}{ Heuristic $\bar{F}^{1}$} & \multicolumn{3}{|c|}{ CPLEX (10 h) } & \multicolumn{5}{|c|}{ Heuristic $^{\bar{F}^{1}}$} \\
\hline$\#$ & $F_{1}^{1}$ & $F_{2}^{1}(\mathrm{k} \$)$ & cpu (s) & $F_{1}^{1}$ & $F_{2}^{1}(\mathrm{k} \$)$ & cpu (s) & Gap1 & Gap2 & $F_{1}^{1}$ & $F_{2}^{1}(\mathrm{k} \$)$ & cpu (s) & $F_{1}^{1}$ & $F_{2}^{1}(\mathrm{k} \$)$ & cpu (s) & Gap1 & Gap2 \\
\hline 1 & 15 & 32.27 & 15162.72 & 15 & 32.27 & 2.35 & 0.00 & 0.00 & 56 & 123.41 & 25162.72 & 60 & 128.82 & 8.53 & 0.07 & 0.04 \\
\hline 2 & 32 & 72.79 & 12387.98 & 32 & 72.79 & 3.14 & 0.00 & 0.00 & - & - & - & 58 & 108.72 & 21.34 & - & - \\
\hline 3 & 18 & 66.78 & 8417.43 & 19 & 66.90 & 5.98 & 0.06 & 0.03 & 44 & 112.01 & 29875.62 & 46 & 113.52 & 16.32 & 0.06 & 0.01 \\
\hline 4 & 16 & 39.26 & 4789.83 & 16 & 39.26 & 8.63 & 0.00 & 0.00 & 40 & 103.18 & 34873.09 & 44 & 107.51 & 18.98 & 0.13 & 0.04 \\
\hline 5 & 24 & 61.87 & 3803.87 & 25 & 62.69 & 3.81 & 0.04 & 0.01 & - & - & - & 50 & 106.72 & 13.21 & - & - \\
\hline 6 & 18 & 60.97 & 19823.45 & 18 & 60.97 & 5.29 & 0.00 & 0.00 & - & - & - & 42 & 90.02 & 15.09 & - & - \\
\hline 7 & 10 & 58.90 & 2113.54 & 10 & 59.72 & 2.51 & 0.00 & 0.01 & 38 & 90.84 & 35219.36 & 38 & 90.84 & 14.11 & 0.00 & 0.00 \\
\hline 8 & 18 & 63.68 & 13923.46 & 18 & 64.76 & 4.25 & 0.00 & 0.02 & - & - & - & 45 & 111.24 & 13.05 & - & - \\
\hline
\end{tabular}




\begin{tabular}{ccccccccc||cccccccc}
9 & 15 & 38.33 & 2893.85 & 16 & 39.74 & 3.39 & 0.07 & 0.04 & 40 & 84.45 & 35194.83 & 40 & 84.45 & 16.93 & 0.00 & 0.00 \\
10 & 9 & 37.81 & 3222.44 & 9 & 37.81 & 1.56 & 0.00 & 0.00 \\
51 & 131.50 & 31356.65 & 55 & 134.77 & 11.36 & 0.05 & 0.02 \\
Ave. & 18 & 53.27 & 7919.61 & 18 & 53.69 & 4.09 & 0.02 & 0.01 & & & & 48 & 107.66 & 14.90 & - & - \\
\hline
\end{tabular}

\begin{tabular}{|c|c|c|c|c|c|c|c|c|c|c|c|c|c|c|c|c|}
\hline \multicolumn{9}{|c|}{ Set 3: $V=30$ vessels, $T=96$ hours } & \multicolumn{8}{|c|}{ Set 4: $V=40$ vessels, $T=168$ hours } \\
\hline \multicolumn{5}{|c|}{ CPLEX (10 h) } & \multicolumn{4}{|c|}{ Heuristic $\bar{F}^{1}$} & \multicolumn{4}{|c|}{ CPLEX $(10 \mathrm{~h})$} & \multicolumn{4}{|c|}{ Heuristic $\bar{F}^{1}$} \\
\hline$\#$ & $F_{1}^{1}$ & $F_{2}^{1}(\mathrm{k} \$)$ & cpu (s) & $F_{1}^{1}$ & $F_{2}^{1}(\mathrm{k} \$)$ & $\mathrm{cpu}(\mathrm{s})$ & Gap1 & Gap2 & $F_{1}^{1}$ & $F_{2}^{1}(\mathrm{k} \$)$ & cpu (s) & $F_{1}^{1}$ & $F_{2}^{1}(\mathrm{k} \$)$ & cpu (s) & Gap1 & Gap2 \\
\hline 1 & - & - & - & 72 & 171.81 & 80.32 & - & - & - & - & - & 84 & 241.61 & 502.43 & - & - \\
\hline 2 & - & - & - & 70 & 166.44 & 48.62 & - & - & - & - & - & 62 & 198.66 & 342.14 & - & - \\
\hline 3 & - & - & - & 75 & 187.92 & 98.37 & - & - & - & - & - & 69 & 220.14 & 278.93 & - & - \\
\hline 4 & - & - & - & 70 & 159.30 & 10.23 & - & - & - & - & - & 62 & 233.56 & 298.34 & - & - \\
\hline 5 & - & - & - & 68 & 155.71 & 89.73 & - & - & - & - & - & 58 & 201.88 & 342.78 & - & - \\
\hline 6 & - & - & - & 78 & 204.03 & 94.74 & - & - & - & - & - & 68 & 171.81 & 392.42 & - & - \\
\hline 7 & - & - & - & 68 & 176.11 & 25.34 & - & - & - & - & - & 98 & 279.20 & 378.04 & - & - \\
\hline 8 & - & - & - & 64 & 172.35 & 15.98 & - & - & - & - & - & 62 & 240.00 & 234.27 & - & - \\
\hline 9 & - & - & - & 60 & 146.04 & 68.89 & - & - & - & - & - & 69 & 220.14 & 409.04 & - & - \\
\hline 10 & - & - & - & 60 & 151.41 & 82.35 & - & - & - & - & - & 71 & 213.16 & 357.93 & - & - \\
\hline Ave. & & - & - & 69 & 169.11 & 61.46 & - & - & & & & 70 & 222.02 & 353.63 & - & - \\
\hline
\end{tabular}

$1 \quad$ Note: Gapl and Gap2 refer to the relative gap of $F_{1}^{1}$ and $F_{2}^{1}$ obtained by CPLEX and Heuristic $\bar{F}^{1}$, respectively. 


\begin{tabular}{|c|c|c|c|c|c|c|c|c|c|c|c|c|c|c|c|c|c|c|c|c|c|c|c|c|}
\hline \multirow[b]{3}{*}{$\#$} & \multicolumn{12}{|c|}{ Set $1: V=10$ vessels, $T=48$ hours } & \multicolumn{12}{|c|}{ Set 2: $V=20$ vessels, $T=72$ hours } \\
\hline & \multicolumn{4}{|c|}{ FCFS rule } & \multicolumn{4}{|c|}{ Productivity rule } & \multicolumn{4}{|c|}{ Gap } & \multicolumn{4}{|c|}{ FCFS rule } & \multicolumn{4}{|c|}{ Productivity rule } & \multicolumn{4}{|c|}{ Gap } \\
\hline & $\tilde{F}_{1}^{10}$ & $\tilde{F}_{2}^{10}$ & $\tilde{F}_{1}^{1 b}$ & $\tilde{F}_{2}^{1 b}$ & $F_{1}^{10}$ & $F_{2}^{10}$ & $F_{1}^{1 b}$ & $F_{2}^{1 b}$ & $G_{1}^{10}$ & $G_{2}^{10}$ & $G_{1}^{1 b}$ & $G_{2}^{1 b}$ & $\tilde{F}_{1}^{10}$ & $\tilde{F}_{2}^{10}$ & $\tilde{F}_{1}^{1 b}$ & $\tilde{F}_{2}^{1 b}$ & $F_{1}^{10}$ & $F_{2}^{10}$ & $F_{1}^{1 b}$ & $F_{2}^{1 b}$ & $G_{1}^{10}$ & $G_{2}^{10}$ & $G_{1}^{1 b}$ & $G_{2}^{1 b}$ \\
\hline 1 & 17 & 40.31 & 15 & 33.56 & 16 & 33.59 & 15 & 32.27 & 0.1 & 0.2 & 0.03 & 0.04 & 75 & 226.66 & 61 & 132.68 & 69 & 148.14 & 60 & 128.82 & 0.08 & 0.53 & 0.02 & 0.03 \\
\hline 2 & 63 & 136.96 & 34 & 76.43 & 36 & 81.52 & 32 & 72.79 & 0.77 & 0.68 & 0.06 & 0.05 & 106 & 178.94 & 60 & 111.98 & 63 & 118.50 & 58 & 108.72 & 0.68 & 0.51 & 0.04 & 0.03 \\
\hline 3 & 32 & 111.69 & 20 & 68.24 & 20 & 70.25 & 19 & 66.90 & 0.58 & 0.59 & 0.03 & 0.02 & 71 & 170.45 & 46 & 118.06 & 48 & 119.20 & 46 & 113.52 & 0.47 & 0.43 & 0.01 & 0.04 \\
\hline 4 & 24 & 54.71 & 16 & 40.05 & 17 & 40.83 & 16 & 39.26 & 0.43 & 0.34 & 0.03 & 0.02 & 64 & 154.99 & 45 & 109.66 & 47 & 113.96 & 44 & 107.51 & 0.38 & 0.36 & 0.02 & 0.02 \\
\hline 5 & 46 & 120.26 & 26 & 64.57 & 27 & 68.33 & 25 & 62.69 & 0.68 & 0.76 & 0.04 & 0.03 & 84 & 152.05 & 51 & 110.99 & 52 & 110.99 & 50 & 106.72 & 0.62 & 0.37 & 0.02 & 0.04 \\
\hline 6 & 28 & 81.01 & 19 & 63.41 & 19 & 62.80 & 18 & 60.97 & 0.49 & 0.29 & 0.03 & 0.04 & 62 & 112.78 & 43 & 91.82 & 45 & 97.22 & 42 & 90.02 & 0.37 & 0.16 & 0.02 & 0.02 \\
\hline 7 & 13 & 75.25 & 10 & 62.71 & 10 & 59.72 & 10 & 59.72 & 0.28 & 0.26 & 0.03 & 0.05 & 51 & 115.55 & 39 & 94.47 & 40 & 96.29 & 38 & 90.84 & 0.27 & 0.2 & 0.02 & 0.04 \\
\hline 8 & 25 & 90.28 & 19 & 68.65 & 18 & 66.38 & 18 & 64.76 & 0.37 & 0.36 & 0.04 & 0.06 & 59 & 142.97 & 46 & 114.58 & 49 & 120.14 & 45 & 111.24 & 0.22 & 0.19 & 0.03 & 0.03 \\
\hline 9 & 22 & 52.65 & 16 & 40.53 & 16 & 40.81 & 16 & 39.74 & 0.32 & 0.29 & 0.03 & 0.02 & 53 & 106.63 & 41 & 86.14 & 43 & 90.36 & 40 & 84.45 & 0.23 & 0.18 & 0.02 & 0.02 \\
\hline 10 & 10 & 41.97 & 9 & 38.57 & 9 & 37.81 & 9 & 37.81 & 0.09 & 0.11 & 0.01 & 0.02 & 69 & 147.17 & 56 & 137.47 & 57 & 140.16 & 55 & 134.77 & 0.21 & 0.05 & 0.01 & 0.02 \\
\hline \multirow[t]{3}{*}{ Ave. } & 28 & 80.51 & 18 & 55.67 & 19 & 56.2 & 18 & 53.69 & 0.41 & 0.39 & 0.03 & 0.04 & 69 & 150.82 & 49 & 110.79 & 51 & 115.50 & 48 & 107.66 & 0.35 & 0.30 & 0.02 & 0.03 \\
\hline & \multicolumn{12}{|c|}{ Set 3: $V=30$ vessels, $T=96$ hours } & \multicolumn{12}{|c|}{ Set 4: $V=40$ vessels, $T=168$ hours } \\
\hline & \multicolumn{4}{|c|}{ FCFS rule } & \multicolumn{4}{|c|}{ Productivity rule } & \multicolumn{4}{|c|}{ Gap } & \multicolumn{4}{|c|}{ FCFS rule } & \multicolumn{4}{|c|}{ Productivity rule } & \multicolumn{4}{|c|}{ Gap } \\
\hline \# & $\tilde{F}_{1}^{10}$ & $\tilde{F}_{2}^{10}$ & $\tilde{F}_{1}^{1 b}$ & $\tilde{F}_{2}^{1 b}$ & $F_{1}^{10}$ & $F_{2}^{10}$ & $F_{1}^{1 b}$ & $F_{2}^{1 b}$ & $G_{1}^{10}$ & $G_{2}^{10}$ & $G_{1}^{1 b}$ & $G_{2}^{1 b}$ & $\tilde{F}_{1}^{10}$ & $\tilde{F}_{2}^{10}$ & $\tilde{F}_{1}^{1 b}$ & $\tilde{F}_{2}^{1 b}$ & $F_{1}^{10}$ & $F_{2}^{10}$ & $F_{1}^{1 b}$ & $F_{2}^{1 b}$ & $G_{1}^{10}$ & $G_{2}^{10}$ & $G_{1}^{1 b}$ & $G_{2}^{1 b}$ \\
\hline
\end{tabular}




\begin{tabular}{|c|c|c|c|c|c|c|c|c|c|c|c|c|c|c|c|c|c|c|c|c|c|c|c|c|}
\hline 1 & 119 & 265.07 & 73 & 175.25 & 84 & 199.30 & 72 & 171.81 & 0.43 & 0.33 & 0.01 & 0.02 & 129 & 353.43 & 85 & 246.44 & 89 & 256.11 & 84 & 241.61 & 0.45 & 0.38 & 0.01 & 0.02 \\
\hline 2 & 111 & 256.15 & 71 & 168.10 & 80 & 189.74 & 70 & 166.44 & 0.39 & 0.35 & 0.02 & 0.01 & 89 & 237.60 & 63 & 200.65 & 64 & 206.61 & 62 & 198.66 & 0.38 & 0.15 & 0.01 & 0.01 \\
\hline 3 & 122 & 296.07 & 76 & 191.68 & 86 & 216.11 & 75 & 187.92 & 0.41 & 0.37 & 0.01 & 0.02 & 81 & 275.06 & 70 & 224.54 & 72 & 231.15 & 69 & 220.14 & 0.12 & 0.19 & 0.01 & 0.02 \\
\hline 4 & 102 & 228.10 & 71 & 162.49 & 78 & 176.82 & 70 & 159.30 & 0.31 & 0.29 & 0.01 & 0.02 & 70 & 283.50 & 63 & 238.23 & 63 & 238.23 & 62 & 233.56 & 0.11 & 0.19 & 0.01 & 0.02 \\
\hline 5 & 97 & 211.89 & 69 & 158.82 & 73 & 168.17 & 68 & 155.71 & 0.32 & 0.26 & 0.02 & 0.02 & 62 & 205.94 & 59 & 205.92 & 59 & 203.90 & 58 & 201.88 & 0.06 & 0.01 & 0.01 & 0.02 \\
\hline 6 & 132 & 327.96 & 80 & 208.11 & 89 & 232.59 & 78 & 204.03 & 0.48 & 0.41 & 0.02 & 0.02 & 79 & 187.51 & 69 & 175.25 & 69 & 175.25 & 68 & 171.81 & 0.14 & 0.07 & 0.02 & 0.02 \\
\hline 7 & 90 & 214.04 & 69 & 177.87 & 70 & 181.39 & 68 & 176.11 & 0.28 & 0.18 & 0.02 & 0.01 & 151 & 447.17 & 101 & 281.99 & 110 & 312.70 & 98 & 279.20 & 0.38 & 0.43 & 0.03 & 0.01 \\
\hline 8 & 73 & 188.10 & 66 & 177.52 & 65 & 175.80 & 64 & 172.35 & 0.12 & 0.07 & 0.03 & 0.03 & 71 & 257.04 & 63 & 242.40 & 63 & 244.80 & 62 & 240.00 & 0.12 & 0.05 & 0.01 & 0.01 \\
\hline 9 & 64 & 156.41 & 61 & 148.96 & 61 & 148.96 & 60 & 146.04 & 0.04 & 0.05 & 0.02 & 0.02 & 81 & 242.51 & 70 & 224.54 & 70 & 224.54 & 69 & 220.14 & 0.15 & 0.08 & 0.01 & 0.02 \\
\hline 10 & 65 & 159.07 & 61 & 152.92 & 61 & 154.44 & 60 & 151.41 & 0.06 & 0.03 & 0.01 & 0.01 & 90 & 252.91 & 72 & 215.29 & 75 & 223.82 & 71 & 213.16 & 0.21 & 0.13 & 0.02 & 0.01 \\
\hline Ave. & 97 & 230.29 & 70 & 172.17 & 75 & 184.33 & 69 & 169.11 & 0.28 & 0.23 & 0.02 & 0.02 & 90 & 274.27 & 71 & 225.53 & 74 & 231.71 & 70 & 222.02 & 0.21 & 0.17 & 0.01 & 0.02 \\
\hline
\end{tabular}


Table 4 Running time under the FCFS and operational productivity rule

\begin{tabular}{|c|c|c|c|c|c|c|c|c|}
\hline \multirow[b]{3}{*}{$\#$} & \multicolumn{4}{|c|}{ Set $1: \mathrm{V}=10$ vessels, $\mathrm{T}=48$ hours } & \multicolumn{4}{|c|}{ Set 2: $\mathrm{V}=20$ vessels, $\mathrm{T}=72$ hours } \\
\hline & \multicolumn{2}{|c|}{ FCFS rule } & \multicolumn{2}{|c|}{ Productivity rule } & \multicolumn{2}{|c|}{ FCFS rule } & \multicolumn{2}{|c|}{ Productivity rule } \\
\hline & CPU(s) & num & $\mathrm{CPU}(\mathrm{s})$ & num & $\mathrm{CPU}(\mathrm{s})$ & num & $\mathrm{CPU}(\mathrm{s})$ & num \\
\hline 1 & 3.28 & 4 & 2.35 & 2 & 7.98 & 5 & 8.53 & 4 \\
\hline 2 & 2.35 & 5 & 3.14 & 3 & 19.82 & 5 & 21.34 & 4 \\
\hline 3 & 1.62 & 5 & 5.98 & 3 & 19.37 & 6 & 16.32 & 4 \\
\hline 4 & 10.29 & 5 & 8.63 & 3 & 15.46 & 4 & 18.98 & 3 \\
\hline 5 & 9.54 & 4 & 3.81 & 2 & 10.84 & 4 & 13.21 & 3 \\
\hline 6 & 7.59 & 4 & 5.29 & 2 & 25.18 & 6 & 15.09 & 5 \\
\hline 7 & 10.32 & 4 & 2.51 & 2 & 10.32 & 3 & 14.11 & 3 \\
\hline 8 & 2.37 & 3 & 4.25 & 2 & 9.72 & 3 & 13.05 & 2 \\
\hline 9 & 5.18 & 6 & 3.39 & 4 & 19.58 & 4 & 16.93 & 3 \\
\hline 10 & 8.25 & 3 & 1.56 & 2 & 12.38 & 4 & 11.36 & 3 \\
\hline \multirow[t]{3}{*}{ Ave. } & 6.08 & 4.3 & 4.09 & 2.5 & 15.07 & 4.4 & 14.90 & 3.4 \\
\hline & \multicolumn{4}{|c|}{ Set 3: $V=30$ vessels, $T=96$ hours } & \multicolumn{4}{|c|}{ Set 4: $V=40$ vessels, $T=168$ hours } \\
\hline & \multicolumn{2}{|c|}{ FCFS rule } & \multicolumn{2}{|c|}{ Productivity rule } & \multicolumn{2}{|c|}{ FCFS rule } & \multicolumn{2}{|c|}{ Productivity rule } \\
\hline$\#$ & $\mathrm{CPU}(\mathrm{s})$ & num & CPU(s) & num & CPU(s) & num & CPU(s) & num \\
\hline 1 & 90.79 & 7 & 80.32 & 6 & 715.32 & 6 & 502.43 & 5 \\
\hline 2 & 60.17 & 5 & 48.62 & 4 & 289.56 & 6 & 342.14 & 5 \\
\hline 3 & 57.24 & 6 & 98.37 & 6 & 315.98 & 7 & 278.93 & 6 \\
\hline 4 & 15.28 & 4 & 10.23 & 3 & 245.26 & 5 & 298.34 & 4 \\
\hline 35 & 82.35 & 5 & 89.73 & 5 & 315.92 & 5 & 342.78 & 3 \\
\hline 6 & 87.59 & 7 & 94.74 & 6 & 222.21 & 5 & 392.42 & 3 \\
\hline 7 & 45.69 & 5 & 25.34 & 3 & 295.55 & 6 & 378.04 & 4 \\
\hline 8 & 35.46 & 5 & 15.98 & 3 & 399.68 & 5 & 234.27 & 3 \\
\hline 9 & 78.66 & 6 & 68.89 & 5 & 458.92 & 5 & 409.04 & 5 \\
\hline 10 & 94.52 & 7 & 82.35 & 5 & 473.26 & 6 & 357.93 & 4 \\
\hline
\end{tabular}




\begin{tabular}{|c|c|c|c|c|c|c|c|}
\hline Ave. & 64.78 & 5.7 & 61.46 & & 373.17 & 353.63 & 4.2 \\
\hline \multicolumn{8}{|c|}{ Table 5 Comparison between solutions to instance \#7 in set 1} \\
\hline \multicolumn{3}{|c|}{ Solutions } & \multicolumn{2}{|c|}{ Difference } & \multicolumn{3}{|c|}{ Ratio } \\
\hline & $F_{1}$ & $F_{2}$ & $\Delta F_{1}$ & $\Delta F_{2}$ & $\Delta F_{1} / F_{1}^{\text {ideal }}$ & $\Delta F_{2} / F_{2}^{\text {nadir }}$ & $\Delta F_{2} / \Delta F_{1}$ \\
\hline & 10 & 58.9 & & & & & \\
\hline & 12 & 56.67 & 2 & 2.23 & 0.2 & 0.04 & 1.11 \\
\hline & 15 & 55.43 & 5 & 3.47 & 0.5 & 0.06 & 0.69 \\
\hline & 18 & 54.02 & 8 & 4.88 & 0.8 & 0.08 & 0.61 \\
\hline & 21 & 52.61 & 11 & 6.29 & 1.1 & 0.11 & 0.57 \\
\hline & 27 & 52.02 & 17 & 6.88 & 1.7 & 0.12 & 0.40 \\
\hline & 33 & 51.42 & 23 & 7.48 & 2.3 & 0.13 & 0.33 \\
\hline Ave: & 19 & 54.44 & 11 & 5.21 & 1.1 & 0.09 & 0.62 \\
\hline
\end{tabular}

